\title{
GLOBULAR CLUSTER SYSTEMS IN BRIGHTEST CLUSTER GALAXIES. III. BEYOND BIMODALITY
}

\author{
William E. Harris ${ }^{1}$, Stephanie M. Ciccone ${ }^{1}$, Gwendolyn M. Eadie ${ }^{1}$, Oleg Y. Gnedin $^{2}$, Douglas Geisler ${ }^{3}$, \\ BARRY ROTHBERG ${ }^{4}$, AND JEREMY BAILIN ${ }^{5}$ \\ ${ }^{1}$ Department of Physics \& Astronomy, McMaster University, Hamilton, ON, Canada; harris@physics.mcmaster.ca, ciccons@mcmaster.ca, eadiegm@mcmaster.ca \\ ${ }^{2}$ Department of Astronomy, University of Michigan, Ann Arbor, MI 48109, USA; ognedin@umich.edu \\ ${ }^{3}$ Departamento de Astronomiá, Universidad de Concepción, Casilla 160-C, Concepción, Chile; dgeisler@astroudec.cl \\ ${ }^{4}$ LBT Observatory, University of Arizona, 933 North Cherry Avenue, Tucson, AZ 85721, USA; dr.barry.rothberg@gmail.com \\ ${ }^{5}$ Department of Physics and Astronomy, University of Alabama, Box 870324, Tuscaloosa, AL 35487-0324, USA; jbailin@ua.edu \\ Received 2016 September 13; revised 2016 November 7; accepted 2016 November 21; published 2017 January 20
}

\begin{abstract}
We present new deep photometry of the rich globular cluster (GC) systems around the Brightest Cluster Galaxies UGC 9799 (Abell 2052) and UGC 10143 (Abell 2147), obtained with the Hubble Space Telescope (HST) ACS and WFC3 cameras. For comparison, we also present new reductions of similar HST/ACS data for the Coma supergiants NGC 4874 and 4889 . All four of these galaxies have huge cluster populations (to the radial limits of our data, comprising from 12,000 to 23,000 clusters per galaxy). The metallicity distribution functions (MDFs) of the GCs can still be matched by a bimodal-Gaussian form where the metal-rich and metalpoor modes are separated by $\simeq 0.8$ dex, but the internal dispersions of each mode are so large that the total MDF becomes very broad and nearly continuous from $[\mathrm{Fe} / \mathrm{H}] \simeq-2.4$ to solar. There are, however, significant differences between galaxies in the relative numbers of metal-rich clusters, suggesting that they underwent significantly different histories of mergers with massive gas-rich halos. Last, the proportion of metal-poor GCs rises especially rapidly outside projected radii $R \gtrsim 4 R_{\mathrm{eff}}$, suggesting the importance of accreted dwarf satellites in the outer halo. Comprehensive models for the formation of GCs as part of the hierarchical formation of their parent galaxies will be needed to trace the systematic change in structure of the MDF with galaxy mass, from the distinctly bimodal form in smaller galaxies up to the broad continuum that we see in the very largest systems.
\end{abstract}

Key words: galaxies: formation - galaxies: star clusters - globular clusters: general

\section{INTRODUCTION}

As a preface to the themes of this paper, it is difficult to improve on the introduction by Geisler et al. (1996), which we quote directly:

\begin{abstract}
"One of the major goals of modern astronomy is an understanding of galaxy formation. An ideal tool for this study would be a witness which was both present at the long-since-vanished first epoch when most galaxies formed, and yet still survives today to tell us its story. In addition, we would like many such witnesses, to corroborate their stories, and we would like them to be easy to find. Enter the globular clusters. They are among our most powerful cosmological probes for investigating this key topic."
\end{abstract}

In this paper we continue an exploration of the globular cluster systems (GCSs) around Brightest Cluster Galaxies (BCGs), the central dominant objects in rich clusters of galaxies. This BCG program extends our earlier work on central giant galaxies at distances within $100 \mathrm{Mpc}$ (Harris et al. 2006; Harris 2009a) outward to richer galaxy-cluster environments and higher BCG luminosities.

Paper I of the current series (Harris et al. 2014) presents new deep photometry obtained with the Hubble Space Telescope (HST) cameras around seven BCGs at distances from 100 to $250 \mathrm{Mpc}$, along with an analysis of the luminosity functions
(LFs) of their GCs. We found the GCLFs to be strikingly similar in all systems, with trends that extend previous analyses for smaller galaxies (Jordán et al. 2007; Villegas et al. 2010) smoothly upward to the largest galaxy sizes known. Paper II (Harris et al. 2016) presents a more comprehensive analysis of the GCS around one of these BCGs, NGC 6166 in Abell 2199, with the focus on its GCS metallicity distribution, spatial distribution, and total GC population.

In this paper, we present similar data for two of the other BCGs in our program, UGC 9799 (Abell 2052) and UGC 10143 (Abell 2147), as well as new data reductions for the two supergiant galaxies NGC 4874 and 4889 in the Coma cluster (Abell 1656), constructed from HST Archive data. In all cases, the photometry reaches very similar depths in absolute magnitude and employs the same color indices, enabling homogeneous comparisons among all the systems. As in Paper II, our focus in this paper is on the metallicity and spatial distributions.

In Section 2 a review of the literature for GC metallicity distributions is presented. In Sections 3 and 4 the target galaxies and the photometric reductions are described. The color-magnitude diagrams for the GC systems are presented in Section 5, and an analysis of the color (metallicity) distributions is presented in Section 6 along with their notable galaxyto-galaxy differences. The spatial distributions are discussed in Section 7. In Sections 8 and 9 we discuss some implications of our findings and present a brief summary.

Our complete photometric data for the five BCGs discussed here and in Paper II can be obtained from the webpage address http://physwww.mcmaster.ca/ harris/BCGdata.html or by request from the first author. 


\section{METALLICITY AND COLOR DISTRIBUTIONS}

A particularly informative feature of globular cluster (GC) populations in galaxies is their metallicity distribution function (MDF). In many galaxies, a long-standing empirical feature of the MDF is its bimodal nature, with a canonical metal-poor (MP) "blue" sequence centered near $\langle[\mathrm{Fe} / \mathrm{H}]\rangle \simeq-1.5$ and a metal-rich (MR) "red" sequence near $\langle[\mathrm{Fe} / \mathrm{H}]\rangle \simeq-0.5$. Many authors have adopted the view that this two-part structure of the MDF is evidence of two major and perhaps distinct starforming epochs in the formation histories of large galaxies, a view that has persisted for many years (e.g., Zepf \& Ashman 1993; Forbes et al. 1997, 2011; Brodie \& Strader 2006; Arnold et al. 2011; Blom et al. 2012b; Brodie et al. 2014; Cantiello et al. 2014; Kartha et al. 2016, among many others). However, bimodal MDFs are not characteristic of the field-halo stars in their parent galaxies, in the few cases where it has been possible to directly compare the GCs and halo stars in the same galaxy (e.g., Harris et al. 2007; Durrell et al. 2010; Rejkuba et al. 2011, 2014; Monachesi et al. 2016). Reconciling this apparent mismatch between field stars and GCs presents an intriguing challenge for quantitative formation modeling.

As is the case for all GC work, the origins of the topic start with the Milky Way. Zinn (1985) clearly established the bimodal nature of the Milky Way GC population, finding that the cluster metallicities coupled closely with systematically different kinematics and spatial distributions for the MP and MR subcomponents. Gradually growing evidence for these two Milky Way subsystems had accumulated in earlier papers (including among others Mayall 1946; Morgan 1956; Baade 1958; Kinman 1959; Marsakov \& Suchkov 1976; Searle \& Zinn 1978; Harris \& Canterna 1979), but it culminated in Zinn's definitive analysis.

For distant galaxies, spectroscopically measured GC metallicities are observationally far more time-consuming to build up, and probing the full three-dimensional kinematics of the halo is out of reach. Instead, GC integrated colors are commonly used as proxies for metallicity, since large samples of GCs can be efficiently measured in this way. For very old and relatively simple stellar systems such as GCs, integrated color is sensitive only to metallicity, while other factors such as mean age or $\mathrm{CNO}$ abundances have only second- or third-order effects. The key empirical question is then how to convert the color distribution function (CDF) of a sample of GCs into its MDF, and whether these different forms are measuring the same phenomenon. The literature on this topic is extensive and continually developing, therefore a full synthesis is probably still premature. However, the issue seems to boil down to two central and only partially related questions.

1. Is the MDF intrinsically bimodal for GC systems?

2. Does the CDF correctly represent the MDF shape after the appropriate transformation?

The answer to the first question, based strictly on spectroscopic evidence, now appears to be that bimodality is common, but that there is no truly universal pattern. As has been emphasized elsewhere (Strader et al. 2011; Usher et al. 2012; Brodie et al. 2014), spectroscopy of significant samples of GCs in many galaxies is needed to confidently go beyond the default assumption of bimodality. The Milky Way GC system is clearly bimodal (see the Appendix of Paper II for a recent version of its MDF based on high-dispersion spectroscopy measures of $[\mathrm{Fe} / \mathrm{H}])$. Several other galaxies now have GC metallicity data constructed from spectrum line strengths, however, and for these, differing results emerge.

In M31, the nearest large galaxy, the GC MDF displays a broad and more uniformly populated distribution that is less easily matched by a bimodal Gaussian form (Barmby et al. 2000; Perrett et al. 2002; Caldwell et al. 2011; Cezario et al. 2013; Caldwell \& Romanowsky 2016), which may reflect the complex and extended growth history of the Galaxy (McConnachie et al. 2009). Other large galaxies with wellpopulated GC systems are rather well described by bimodal, spectroscopic MDFs: these include NGC 5128 (Woodley et al. 2010), M81 (Ma et al. 2013), NGC 4472 (Strader et al. 2007), NGC 4594 (Alves-Brito et al. 2011), NGC 3115 (Arnold et al. 2011; Brodie et al. 2012), and eight other large normal ellipticals (Foster et al. 2010; Usher et al. 2012). However, notable galaxy-to-galaxy differences appear in the degree of overlap between the MP and MR "modes" and their internal dispersions. Spectroscopically based evidence of trimodality or simply a more uniform $[\mathrm{Fe} / \mathrm{H}]$ distribution is indicated for M87 (Strader et al. 2011), NGC 4494 (Usher et al. 2012), and perhaps NGC 4365 (Blom et al. 2012a; Chies-Santos et al. 2012b) as well as M31.

The answer to the second question-how well the CDF represents the MDF-strongly depends on which color index is being used. Many indices from near-UV through to near-IR have now been tested and compared. Several discussions have claimed that a unimodal MDF is capable of being converted into a bimodal CDF if the transformation is sufficiently nonlinear (e.g., Yoon et al. 2006, 2011a, 2011b; Cantiello \& Blakeslee 2007; Chies-Santos et al. 2012a; Kim et al. 2013; Chung et al. 2016). As a numerical exercise, this claim is certainly true, although the continuing issue with these discussions is that they rely very heavily on single-stellarpopulation (SSP) theoretical modeling to develop translation curves from $[\mathrm{Fe} / \mathrm{H}]$ to a given color index. These model curves usually have quite complex shapes, and the various available SSP libraries show notable disagreements (e.g., Alves-Brito et al. 2011; Peacock et al. 2011; Brodie et al. 2012; Chung et al. 2016).

By contrast, empirically based transformations from metallicity to color that do not rely heavily on modeling tend to be much more nearly linear and thus to yield CDFs that resemble the intrinsic MDFs rather well (e.g., Barmby et al. 2000; Peng et al. 2006; Spitler et al. 2008; Fan et al. 2010; Foster et al. 2010; Sinnott et al. 2010; Peacock et al. 2011; Brodie et al. 2012, 2014; Usher et al. 2012, 2015; Cantiello et al. 2014; Vanderbeke et al. 2014; Caldwell \& Romanowsky 2016; Sakari \& Wallerstein 2016). SSP theoretical models, however, are useful for comparing the relative metallicity sensitivity and linearity of different color indices. The most effective indices include $(V-K),(B-I),\left(g^{\prime}-i^{\prime}\right),\left(g^{\prime}-I\right),\left(g^{\prime}-K\right)$, or $\left(C-T_{1}\right.$ ) (Barmby et al. 2000; Harris et al. 2006; Cantiello \& Blakeslee 2007; Kundu \& Zepf 2007; Spitler et al. 2008; Fan et al. 2010; Foster et al. 2010; Sinnott et al. 2010; Blakeslee et al. 2012; Forte et al. 2013). These indices combine high sensitivity to metallicity with modest degrees of nonlinearity. However, a large fraction of the CDFs available in the literature have been measured in $(V-I)$ (which is less sensitive to metallicity) or $\left(g^{\prime}-z^{\prime}\right)$ (which is more nonlinear than others listed above) (e.g., Gebhardt \& Kissler-Patig 1999; Larsen et al. 2001; Peng et al. 2006; Villegas et al. 2010). A diagnostic index that has recently gained more frequent use is the $\mathrm{Ca}$ 
triplet line strength, which correlates almost linearly with [Fe/ $\mathrm{H}]$ in the range $[\mathrm{Fe} / \mathrm{H}] \lesssim-0.5$ (Brodie et al. 2012; Sakari \& Wallerstein 2016). At the highest metallicities, CaT may become less sensitive, but any change in slope above [Fe/ $\mathrm{H}] \simeq-0.5$ will not be able to generate the intermediatemetallicity "valley" at $[\mathrm{Fe} / \mathrm{H}] \simeq-1$ between the normal MP and MR modes.

Interestingly, Usher et al. (2015) discuss evidence that different galaxies may have different color-to-metallicity conversions. Cantiello \& Blakeslee (2007) and Forte et al. (2013) note that for a given galaxy, every color index should yield the same MDF if the transformations are correct. So far, applying this self-consistency test in practice has rarely been possible.

A useful conclusion for the present seems to be that CDFs reflect the intrinsic shapes of the MDFs, if the color indices being used are chosen well. The extensive literature that reveals clearly bimodal CDFs for many galaxies therefore continues to be important (e.g., Geisler et al. 1996; Neilsen \& Tsvetanov 1999; Kundu \& Whitmore 2001; Larsen et al. 2001; Rhode \& Zepf 2004; Bassino et al. 2006; Peng et al. 2006; Harris 2009a, 2009b; Faifer et al. 2011; Cantiello et al. 2014; Jennings et al. 2014; Kartha et al. 2014, among many others). At the same time, some galaxies are better described as trimodal, unimodal, or simply broad without matching a simple Gaussian-type model (for specific examples, see Larsen et al. 2001, 2005; Peng et al. 2006; Kundu \& Zepf 2007; Blom et al. 2012b; Usher et al. 2012). A valid theoretical model for GC formation in the larger context of galaxy formation must be able to deal with this diversity of outcomes.

Starting with either the CDF or MDF, the first empirical question is simply to establish how many components or "modes" are present regardless of shape, and how similar these might be to the Milky Way; or (alternately) whether a bimodal deconstruction is justified in the first place. The first mention of a specifically Gaussian shape for these modes that we are aware of is in Zinn (1985). Zepf \& Ashman (1993) introduced a mixture-modeling numerical code (the since-popular KMM package) to make objective tests of unimodality (the null hypothesis) versus multimodality, using the CDFs for the two giant ellipticals NGC 4472 and 5128 as testbed cases. The authors concluded that their CDFs are clearly bimodal. A Gaussian model was implicitly used for fitting the components, and, more or less by default, this quickly became the norm for later studies. Just a few years later, the "bimodal Gaussian" model was already rather firmly established in the literature of the subject (e.g., Geisler et al. 1996).

Gebhardt \& Kissler-Patig (1999) used various nonparametric tests to establish the Gaussian bimodal form more strongly, although of necessity they were forced to work with the rather metallicity-insensitive color index $(V-I)$ that dominated the available data at the time. Muratov \& Gnedin (2010) discuss different indicators of either Gaussianity or bimodality within the context of their GMM fitting code, noting that a unimodal but asymmetric MDF will often favor a bimodal Gaussian fit. The bimodal-Gaussian form continues to be widely used simply because it continues to match the CDFs and MDFs rather accurately in large numbers of galaxies of all types, sizes, and environments as the quality and internal precision of the data have steadily increased. Nevertheless, there is not yet any a priori astrophysical reason to say that the MP and MR components should be specifically Gaussian. GC formation
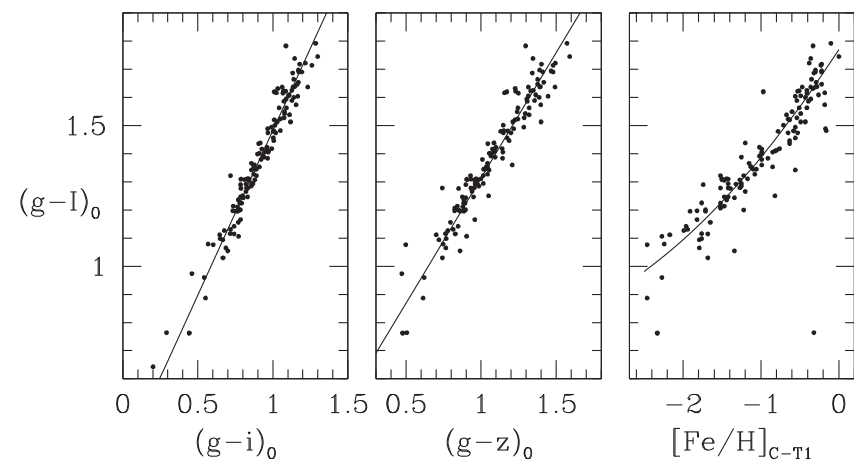

Figure 1. Left panel: $(g-I)_{0}$ vs. $(g-i)_{0}$ for clusters in NGC 5128. Middle panel: $(g-I)_{0}$ vs. $(g-z)_{0}$ for the same clusters. Right panel: $(g-I)_{0}$ vs. $[\mathrm{Fe} / \mathrm{H}]$ for the same clusters. The equations for the interpolation lines are given in the text: in the right panel, the solid line is Equation (1) in the text.

models within the context of their parent galaxies are not yet advanced enough to make predictions for the shape of the MDF at that level of precision (e.g., Li \& Gnedin 2014). Thus the main purpose of these numerical model fits is to conveniently characterize the first-order features of the MDF: the mean metallicities of the modes (however many there are), their widths (intrinsic metallicity spread), and the metallicity separation between modes.

In the present survey of GCSs in BCGs, we use the color index $(F 475 W-F 814 W) \simeq(g-I)$ (from here on we drop the accents on the SDSS indices). In the following discussion it will be useful to have a calibration of this index versus cluster metallicity $[\mathrm{Fe} / \mathrm{H}]$. To do this, we would ideally need to have GC photometry of the same clusters in both the Kron-Cousins and SDSS systems, in addition to spectroscopically based metallicity measurements. At present, there are no ideal solutions to that problem. Galaxies satisfying all three of these criteria are rare; in principle the Milky Way GC databases could be used, but cluster-to-cluster foreground reddenings differ strongly, the published SDSS indices (Vanderbeke et al. 2014) show considerable scatter versus metallicity, and the variety of studies from which the UBVRI indices were derived are completely different from the SDSS survey, so that aperture-size mismatches are significant. Similar problems affect the M31 GC sample. The best option at the present time for developing a $(g-I)$ transformation is likely to be from the nearby early-type giant galaxy NGC 5128: here, UBVRI photometry is available from Peng et al. (2004), griz photometry from Sinnott et al. (2010), and $[\mathrm{Fe} / \mathrm{H}]$ values derived through $\left(C-T_{1}\right)$ from Woodley et al. (2010); these $[\mathrm{Fe} / \mathrm{H}]$ values are in turn well correlated with the Sloan-system spectroscopic index [MgFe]' (see Woodley et al.). We have extracted the GCs in common from these three catalogs, with the results shown in Figure 1. The great majority of these GCs lie well outside the central few kiloparsecs of NGC 5128 and thus are unaffected by the well-known dust lane. We have therefore applied only the foreground reddening of the Galaxy, for which we adopt $E_{g-I}=2.2 E_{B-V}=0.25$ (Cardelli et al. 1989) to obtain the intrinsic colors. We note, however, that the UBVRI measurements were done on $3^{\prime \prime}$ aperture diameters corrected to $14^{\prime \prime}$ through median curves of growth (Peng et al. 2004), while the griz measures were done through 7!"6 apertures (Sinnott et al. 2010), which means that a small 
aperture mismatch may exist here as well that affects the zeropoint of $(g-I)$.

The first two panels of Figure 1 show the correlations between $(g-i)_{0}$ and $(g-z)_{0}$ versus $(g-I)_{0}$. These correlations rely purely on the photometric data independently of $[\mathrm{Fe} / \mathrm{H}]$ estimates. Simple linear relations derived from direct least-squares fits are

$$
\begin{aligned}
(g-i)_{0} & =(-0.268 \pm 0.024)+(0.856 \pm 0.018)(g-I)_{0}, \\
(g-z)_{0} & =(-0.475 \pm 0.042)+(1.123 \pm 0.030)(g-I)_{0} .
\end{aligned}
$$

The $(g-i)_{0}$ versus $(g-I)_{0}$ relation is more tightly defined and valid over a wider range of colors than $(g-z)_{0}$ versus $(g-I)_{0}$. Both $(g-i)$ and $(g-I)$ do well as metallicity indicators, but $(g-I)$ is slightly more sensitive and takes good advantage of the broadband $H S T$ filter system. The $(g-z)$ index in turn is slightly more sensitive than $(g-I)$, but a noticeable nonlinearity remains between them. It should be noted again (see above) that the $g$ and $I$ photometric data come from two different observational programs and therefore do not have the internal homogeneity that would normally be desired, so that any error in the zero-point of the $(g-I)$ scale is hard to assess at present. Fortunately, the slope and curvature of the relations are more important for the purposes here than the absolute values of $[\mathrm{Fe} / \mathrm{H}]$.

The third panel of Figure 1 connects $(g-I)_{0}$ with $[\mathrm{Fe} / \mathrm{H}]$. A modestly nonlinear quadratic relation accounting for scatter in both axes is

$$
(g-I)_{0}=1.770+0.428[\mathrm{Fe} / \mathrm{H}]+0.045[\mathrm{Fe} / \mathrm{H}]^{2},
$$

which is plotted in Figure 1(c). We recognize that this proposed calibration is only temporary; in particular, the zero-point depends on the accuracy of the separate zero-points of $g$ and $I$ from two different observational programs and thus has a higher degree of uncertainty than usual. The conversion of $(g-I)$ to $[\mathrm{Fe} / \mathrm{H}]$ can be greatly solidified once larger numbers of high-quality spectroscopically based $[\mathrm{Fe} / \mathrm{H}]$ values become available for GC systems outside the Local Group particularly, where aperture-size corrections on the photometry become unimportant.

\section{TARGET GALAXIES}

In this paper, we present new photometry for the GC populations around UGC 9799 and UGC 10143. For comparison with these and the other BCGs in our program, we also include NGC 4874 and NGC 4889 in the Coma cluster. In all cases, the main data are from the ACS Wide Field Camera on board HST, with identical filters and similar exposure time. Here, we briefly summarize basic features of these galaxies and the Abell clusters they dominate.

UGC 9799 is the central and brightest galaxy in Abell 2052 at a distance $d=150 \mathrm{Mpc}$ (for $H_{0}=70 \mathrm{~km} \mathrm{~s}^{-1} \mathrm{Mpc}^{-1}$ ). As in Paper I, we adopt an apparent distance modulus $(m-M)_{I}=35.95$ and foreground reddening (from NED, following Schlafly \& Finkbeiner 2011) $E_{B-V}=0.037$. The GC system around UGC 9799 was first detected by Harris et al. (1995) through deep CFHT imaging. A reproduction of our ACS/WFC field is shown in Figure 2.

The core of UGC 9799 shows clear evidence of gas and (modest) nuclear activity. As a radio source, it is $3 \mathrm{C} 317$, and Chandra observations reveal a compact core X-ray source
(Donato et al. 2004; Balmaverde et al. 2006). At the center, GALEX ultraviolet profiles and infrared excess lead to a deduced present-day star formation rate of $\sim 1 M_{\odot} \mathrm{yr}^{-1}$ (Hicks et al. 2010; Hoffer et al. 2012), while nuclear optical emission lines and a small star-forming filament visible in the near-UV further confirm star-forming activity (Martel et al. 2002; Buttiglione et al. 2010); the filament is also visible in our $F 475 W$ image. There is presumably a central supermassive black hole, but only an upper limit of $4.6 \times 10^{9} M_{\odot}$ exists for it (Dalla Bontà et al. 2009). The larger-scale distribution of hot $\mathrm{X}$-ray gas in A2052 has recently been discussed by Blanton et al. (2011) and Machado \& Lima Neto (2015); the hot gas shows much substructure (bubbles, shocks), which is indicative of previous AGN activity and/or merging of galaxies.

UGC 10143 is the central giant in A2147, at $d=154 \mathrm{Mpc}$; and A2147 is part of the Hercules supercluster along with A2151 and A2152 (Barmby \& Huchra 1998). We adopt $(m-M)_{I}=35.99$ and $E_{B-V}=0.031$. The BCG is radio quiet and has a deduced central star formation rate consistent with zero from its lack of UV or infrared signature (Hoffer et al. 2012). Close inspection of our images shows a smooth, featureless optical core with no indications of peculiarities. The GC system around UGC 10143 was first detected by Blakeslee (1999) through ground-based imaging and surface brightness fluctuation (SBF) analysis. A reproduction of our ACS/WFC field is shown in Figure 3.

According to Tovmassian \& Andernach (2012), A2147 is not a Bautz-Morgan class I cluster since the luminosity difference in $M_{K}$ between its first- and second-ranked galaxies is only 0.2 mag (the second-ranked member is PGC056770, which lies $180 \mathrm{kpc}$ to the south of UGC 10143). However, the overall cluster richness, the velocity dispersion, and the moderately low peculiar motion of the BCG are all typical of BM I clusters, therefore the classification remains a bit ambiguous.

The giants NGC 4874 and NGC 4889 are the dominant galaxies in the rich and well-known Coma cluster (A1656), for which we adopt $d=100 \mathrm{Mpc},(m-M)_{I}=35.02$, and $E_{B-V}=0.01$. Reproductions of the NGC 4889 and NGC 4874-F2 fields from the ACS/WFC camera are shown in Figures 4 and 5. NGC 4874 is surrounded by a handful of smaller satellite galaxies (see Figure 1 of Cho et al. 2016, for their identification numbers), but as we show below, these do not contribute noticeably to the overall GC population, with the exception of a small excess around NGC 4873 (at left center in Figure 5). Although NGC 4874 and 4889 have similar $V$-band luminosities, NGC 4874 is clearly the one lying at or near the center of the Coma potential well (as defined by the intracluster X-ray gas) and has a cD-type envelope. By contrast, NGC 4889 resembles a structurally more normal elliptical, but with a supergiant-level luminosity. In our images, both have smooth featureless isophotes all the way inward to the Galaxy center. The GC populations around these galaxies were first detected 30 years ago by Harris (1987) and Thompson \& Valdes (1987) through deep imaging with the CFHT. Later, ground-based imaging by Blakeslee \& Tonry (1995) and Marín-Franch \& Aparicio (2002) including SBF techniques verified that both galaxies had rich GC systems. Harris et al. (2009) presented homogeneous photometry of the GC systems in five Coma ellipticals including the two supergiants, all with data from the HST WFPC2 camera. These reached deep enough to gauge the GC luminosity function turnover point and to obtain useful 


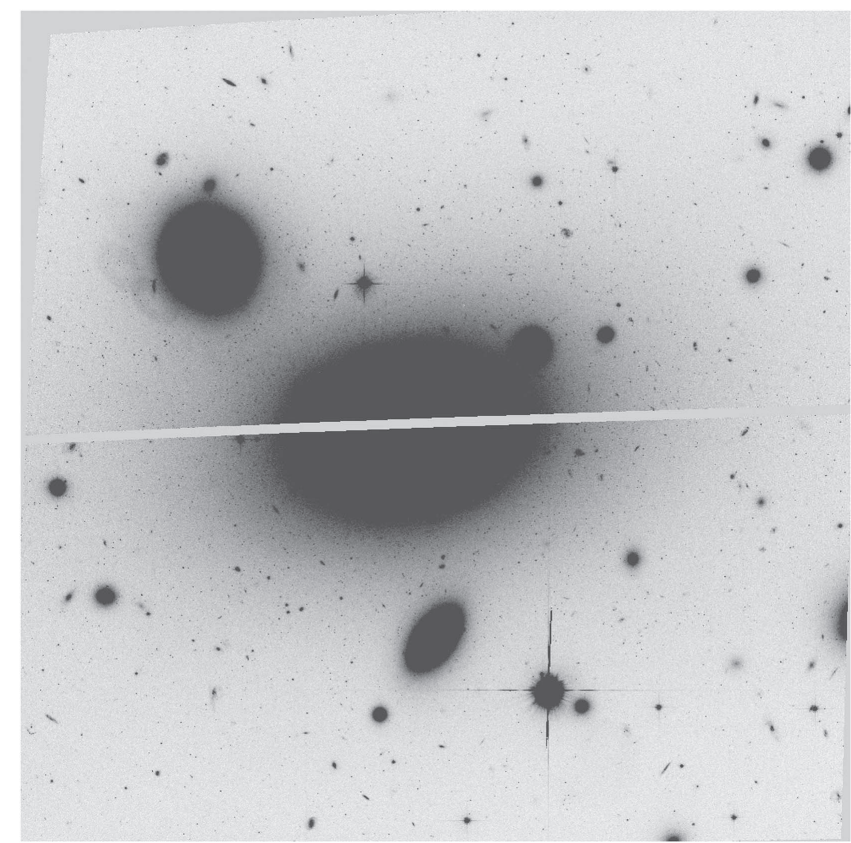

Figure 2. ACS/WFC field centered on UGC 9799. The field of view is roughly $3 ! 4$ across.

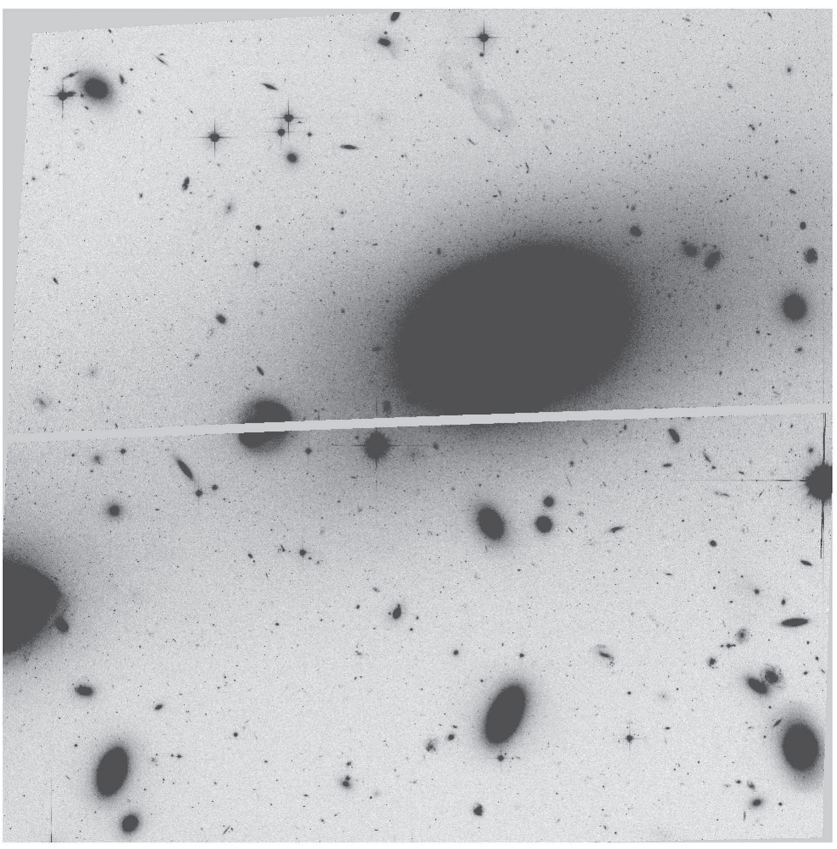

Figure 3. ACS/WFC field centered on UGC 10143.

values for the GC specific frequencies in the galaxies, but color indices ( $V-I$ in this case) were not precise enough to clearly resolve the $\mathrm{CDF}$ and determine whether these systems fall within the conventional bimodal pattern.

More recently, Peng et al. (2011) discussed the distribution of GCs throughout the Coma cluster using the HST/ACS Coma Cluster Treasury Survey imaging. Their analysis shows that NGC 4874 is essentially at the center of the GC distribution in Coma and that its own GC spatial profile makes a clear transition to a newly discovered intragalactic cluster (IGC) population, which becomes dominant beyond a projected radius of $\sim 300 \mathrm{kpc}$. In the CDF, two modes (MP and MR) are clearly present, and the MP mode is much more dominant for

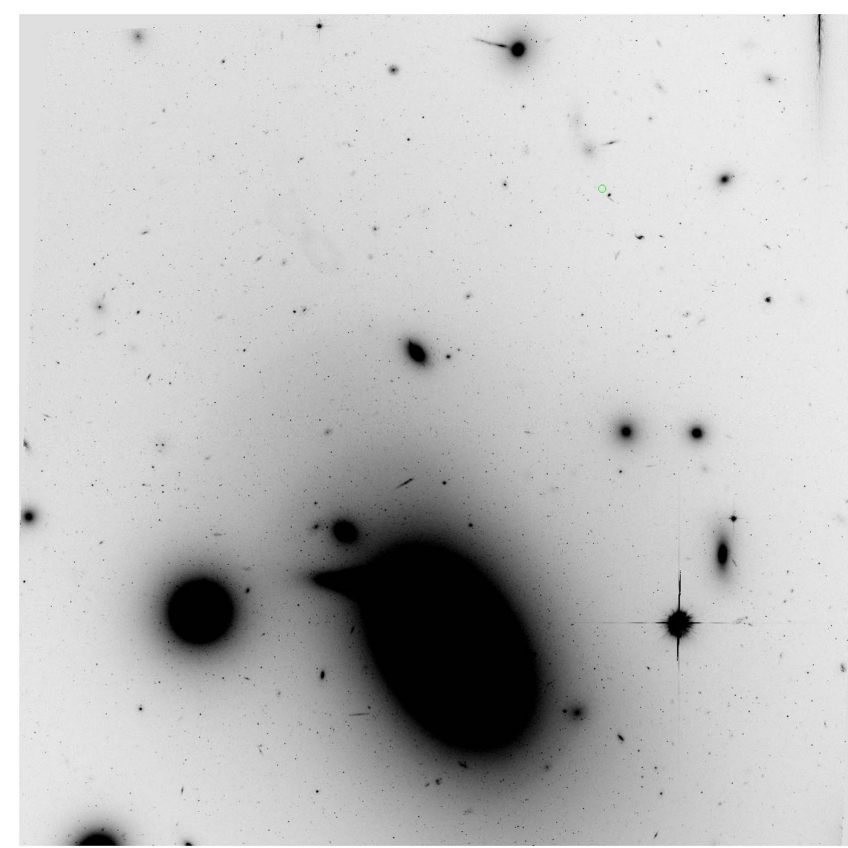

Figure 4. ACS/WFC field containing NGC 4889.

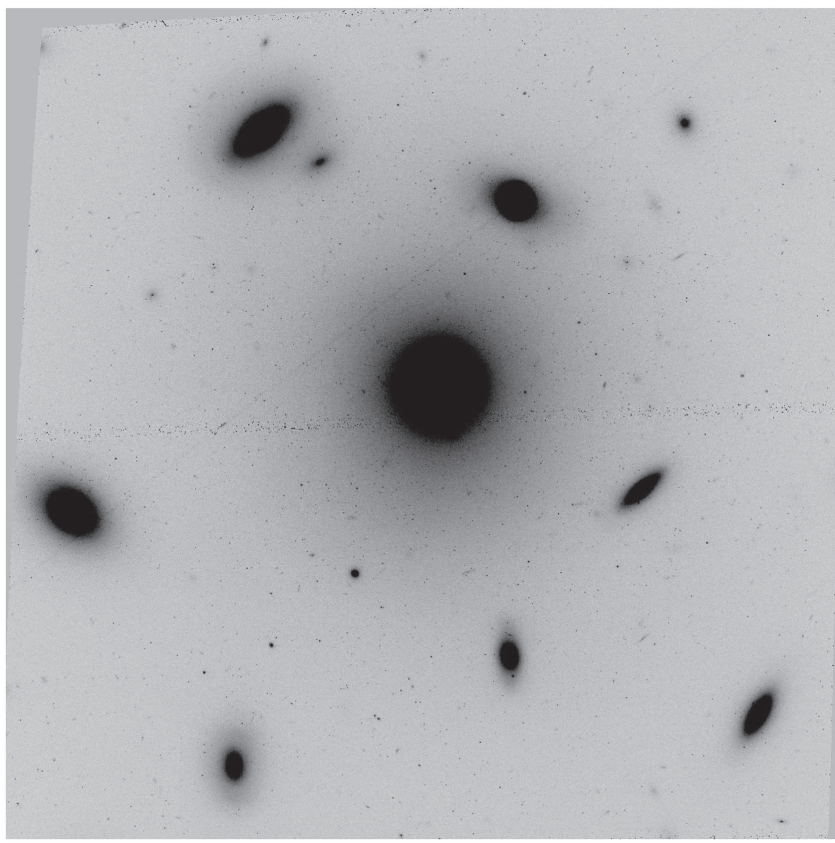

Figure 5. ACS/WFC field (F2) centered on NGC 4874.

the IGC. Color-magnitude diagrams for the GC populations around both NGC 4874 and 4889 from HST ACS imaging are presented and briefly discussed by Lee \& Jang (2016a), while Cho et al. (2016) complete a more comprehensive discussion specifically for the NGC 4874 system, now including threecolor $(g, I, H)$ photometry.

A structural feature held in common by all four of the Galaxy clusters discussed here (Coma, A2052, A2147, and A2199 from Paper II) is a prominent X-ray halo gas component. Of 60 nearby clusters listed by Edwards et al. (2007) that were selected from the NOAO Fundamental Plane Survey, these four rank among the highest in X-ray luminosity, but not all of them have strong cooling flows or central optical 
Table 1

BCG Parameters

\begin{tabular}{lcccccr}
\hline \hline Galaxy & Cluster & $(m-M)_{I}$ & $d$ & $M_{K}$ & $\begin{array}{c}L_{X} \\
\left(10^{44}\right.\end{array}$ & $R_{c}$ \\
& & & $(\mathrm{Mpc})$ & & $\left.\mathrm{erg} \mathrm{s}^{-1}\right)$ & $(\mathrm{kpc})$ \\
\hline NGC 4874 & A1656 & 35.02 & 100 & -26.1 & 3.98 & $(0)$ \\
NGC 4889 & A1656 & 35.02 & 100 & -25.6 & 3.98 & 169 \\
NGC 6166 & A2199 & 35.60 & 130 & -25.7 & 1.90 & 7 \\
UGC 9799 & A2052 & 35.95 & 150 & -25.5 & 1.33 & 38 \\
UGC 10143 & A2147 & 35.99 & 154 & -24.9 & 1.66 & 82 \\
\hline
\end{tabular}

emission. IGC populations have clearly been established to date only for Virgo and Coma (Peng et al. 2011; Durrell et al. 2014), and more tentatively in Abell 1689 (Alamo-Martínez et al. 2013), Abell 1185 (West et al. 2011), and Abell 2744 (Lee \& Jang 2016b), and it is not yet known how well their presence correlates with hot halo gas. However, the X-ray halos demonstrate that the BCGs studied here all reside in very massive potential wells defined by their surrounding clusters. The virial masses of these clusters as confirmed through galaxy velocity dispersions, X-ray gas temperature, or weak lensing are typically $M_{200} \sim 10^{14}-10^{15} M_{\odot}$ (e.g., Blanton et al. 2003; Łokas et al. 2006; Kubo et al. 2007; Wen et al. 2010; Wojtak \& Lokas 2010; Falco et al. 2014).

In Table 1 we summarize some of the fiducial properties of the BCGs discussed in this paper. The last column gives the projected distance $R_{c}$ of each BCG from the center of its Abell cluster (see the references cited above).

\section{PHOTOMETRIC REDUCTIONS}

Imaging for our program was made with the $F 475 \mathrm{~W}$ and $F 814 W$ filters. The resulting color index in the native filters, $(F 475 W-F 814 W)$, is close to standard $(g-I)$ and is both metallicity-sensitive and nearly linearly correlated with metallicity (see Section 2). The magnitude scale we adopt here, as in previous work (Harris 2009a, and Papers I and II), is on the VEGAMAG system.

The raw imaging data for UGC 9799 and 10143 are from HST program GO-12238 (PI Harris). Design parameters for this program are summarized in Paper I; full details of the photometric data reductions are laid out in Paper II, and we follow the same procedures here. For these two galaxies, ACS/ WFC exposures were taken roughly centered on the BCG, while Parallel exposures with WFC3/UVIS (in the same filters) were taken simultaneously to give an offset field located in the outskirts of the galaxy cluster.

From the * flc raw image files provided in the HST Archive we constructed a single combined image in each filter with stsdas/multidrizzle. We used SourceExtractor (Bertin \& Arnouts 1996) to detect candidate objects in each field, and preliminarily to reject nonstellar objects. Then we used the normal sequence of steps in iraf/daophot/allstar (Stetson 1987) to complete the photometry from point-spread function (PSF) fitting and to again reject nonstellar objects from the goodnessof-fit $\chi$ parameter and the internal measurement uncertainties in each filter. In all cases, the candidate GCs we searched for are expected to be starlike in structure for galaxies more distant than $d \gtrsim 80 \mathrm{Mpc}$ (see Papers I and II as well as Harris 2009a). This is an important advantage for our purposes, because it facilitates removing the vast majority of the field
Table 2

Exposure Times and Completeness Parameters

\begin{tabular}{|c|c|c|c|c|c|c|}
\hline Galaxy & $\begin{array}{c}\text { GO } \\
\text { Program }\end{array}$ & Detector & Filter & $t(\mathrm{~s})$ & $m_{0}$ & $\alpha$ \\
\hline \multirow[t]{4}{*}{ UGC 9799} & 12238 & $\mathrm{ACS} / \mathrm{WFC}$ & $F 475 W$ & 7977 & 29.60 & 3.5 \\
\hline & & & $F 814 W$ & 5253 & 28.13 & 3.5 \\
\hline & & WFC3 & $F 475 W$ & 8041 & 29.30 & 3.0 \\
\hline & & & $F 814 W$ & 5343 & 27.85 & 2.7 \\
\hline \multirow{4}{*}{$\begin{array}{l}\text { UGC } \\
10143\end{array}$} & 12238 & $\mathrm{ACS} / \mathrm{WFC}$ & $F 475 W$ & 10726 & 29.45 & 3.5 \\
\hline & & & $F 814 W$ & 5262 & 27.95 & 3.3 \\
\hline & & WFC3 & $F 475 W$ & 10856 & 29.20 & 3.0 \\
\hline & & & $F 814 W$ & 5352 & 27.30 & 3.3 \\
\hline \multirow[t]{2}{*}{ NGC 4889} & 11711 & $\mathrm{ACS} / \mathrm{WFC}$ & $F 475 W$ & 4770 & 29.20 & 2.6 \\
\hline & & & $F 814 W$ & 9960 & 28.00 & 3.2 \\
\hline \multirow{2}{*}{$\begin{array}{l}\text { NGC } \\
\qquad 4874-F 1\end{array}$} & 10861 & $\mathrm{ACS} / \mathrm{WFC}$ & $F 475 W$ & 2677 & 28.30 & 2.6 \\
\hline & & & $F 814 W$ & 1400 & 27.10 & 3.2 \\
\hline \multirow{2}{*}{$\begin{array}{l}\text { NGC } \\
\quad 4874-F 2\end{array}$} & 11711 & $\mathrm{ACS} / \mathrm{WFC}$ & $F 475 W$ & 2394 & 28.60 & 2.6 \\
\hline & & & $F 814 W$ & 10425 & 28.00 & 3.2 \\
\hline \multirow{2}{*}{$\begin{array}{l}\text { NGC } \\
\quad 4874-\mathrm{F} 3\end{array}$} & 12918 & ACS/WFC & $F 475 W$ & 2568 & 28.30 & 2.6 \\
\hline & & & $F 814 W$ & 1400 & 26.90 & 3.2 \\
\hline
\end{tabular}

contamination, which is dominated by faint very small but resolved background galaxies.

Last, artificial-star tests were separately run with daophot/ addstar to quantify the detection completeness fraction as a function of magnitude, $f(m)$, for each target field and filter. The $f(m)$ data were fit to a smooth curve of the form

$$
f(m)=\frac{1}{1+e^{\alpha\left(m-m_{0}\right)}},
$$

as defined in Paper II. Here $m_{0}$ represents the $50 \%$ completeness level and $\alpha$ the steepness of falloff as the curve passes through $m_{0}$. Nominally, $f$ is also a function of the background light intensity and therefore of the projected galactocentric distance. However, for these distant and rather diffuse BCGs, at $R \gtrsim 15^{\prime \prime}$ the surface brightness has already fallen to a low enough level that the radial dependence beyond that radius is negligible (see Paper I for a discussion). In our following analysis, we do not use any of the raw data within $15^{\prime \prime}$ of the Galaxy centers. More detailed descriptions of the procedures and examples can be found in Paper II and Harris (2009a). In Table 2 we list in successive columns the galaxy name, GO program ID from the HST Archive, camera, filter name, total exposure time, and completeness function parameters.

\section{COLOR-MAGNITUDE DIAGRAMS}

In Figure 6 we show the distribution of measured starlike objects brighter than $F 814 W=27.0$ in the UGC 9799 ACS and WFC3 fields. In this magnitude range, as shown in Paper II, almost all of these objects are expected to be GCs. In the ACS field, two smaller companion galaxies are visible as separate compact groups of GCs of their own: these are PGC054528 (in the upper left, marked out by a circle of $15^{\prime \prime}$ radius), and PGC05421 (below UGC 9799 and marked out by a $r=10^{\prime \prime}$ circle). In the WFC3 field, the galaxy in the upper left with an obvious GC population of its own is PGC054533, 

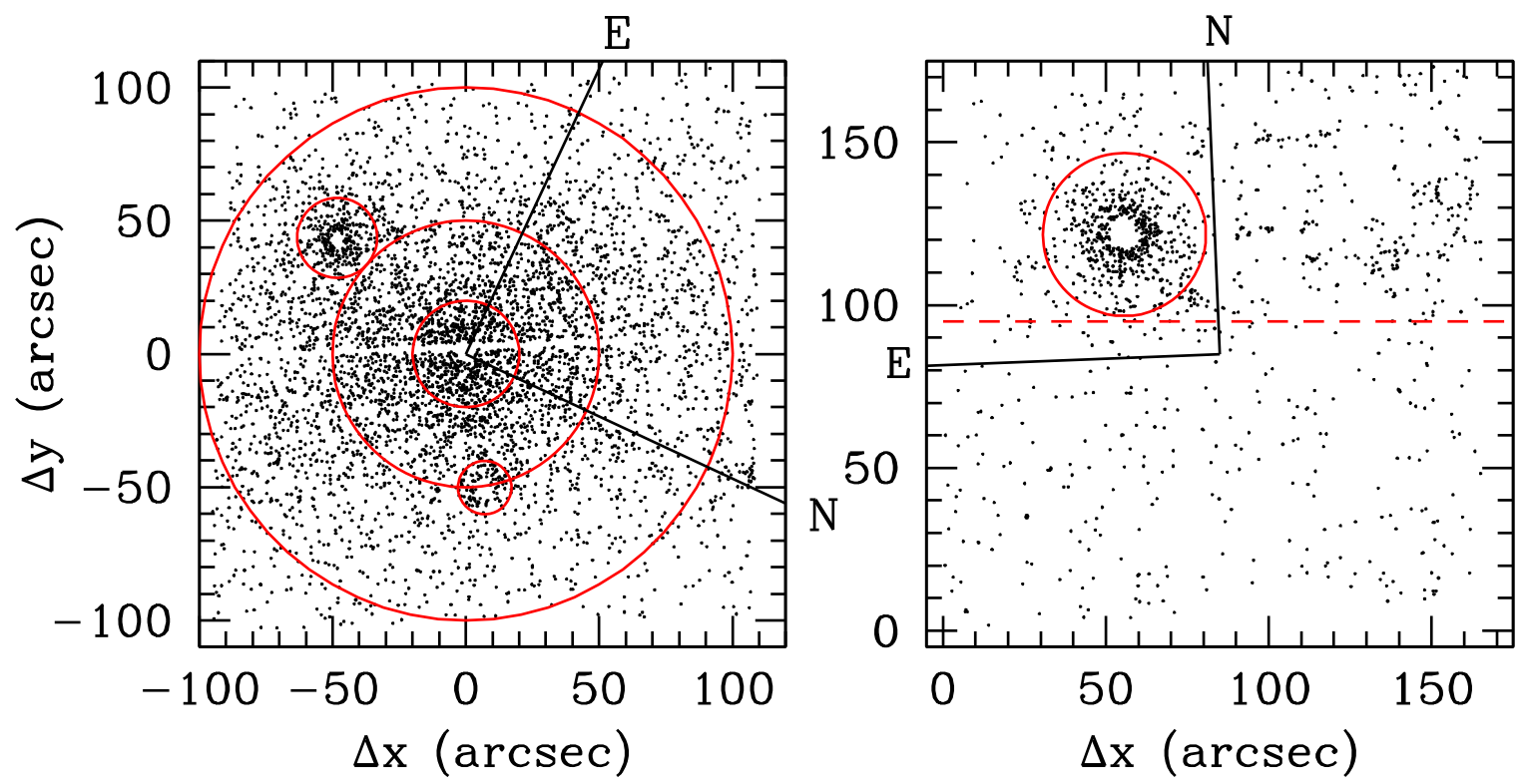

Figure 6. Locations of the measured starlike objects brighter than $F 814 \mathrm{~W}=27.0$ in the ACS field centered near UGC 9799 (left panel) and in the Parallel WFC 3 field (right panel). Fiducial directions on the sky (north, east) are marked in both panels. Small red circles mark smaller companion galaxies with GC populations of their own, as listed in the text. For WFC3, the red dashed line indicates the border between contaminating small galaxies (above the line) and a cleaner sample of IGC GCs (below the line).
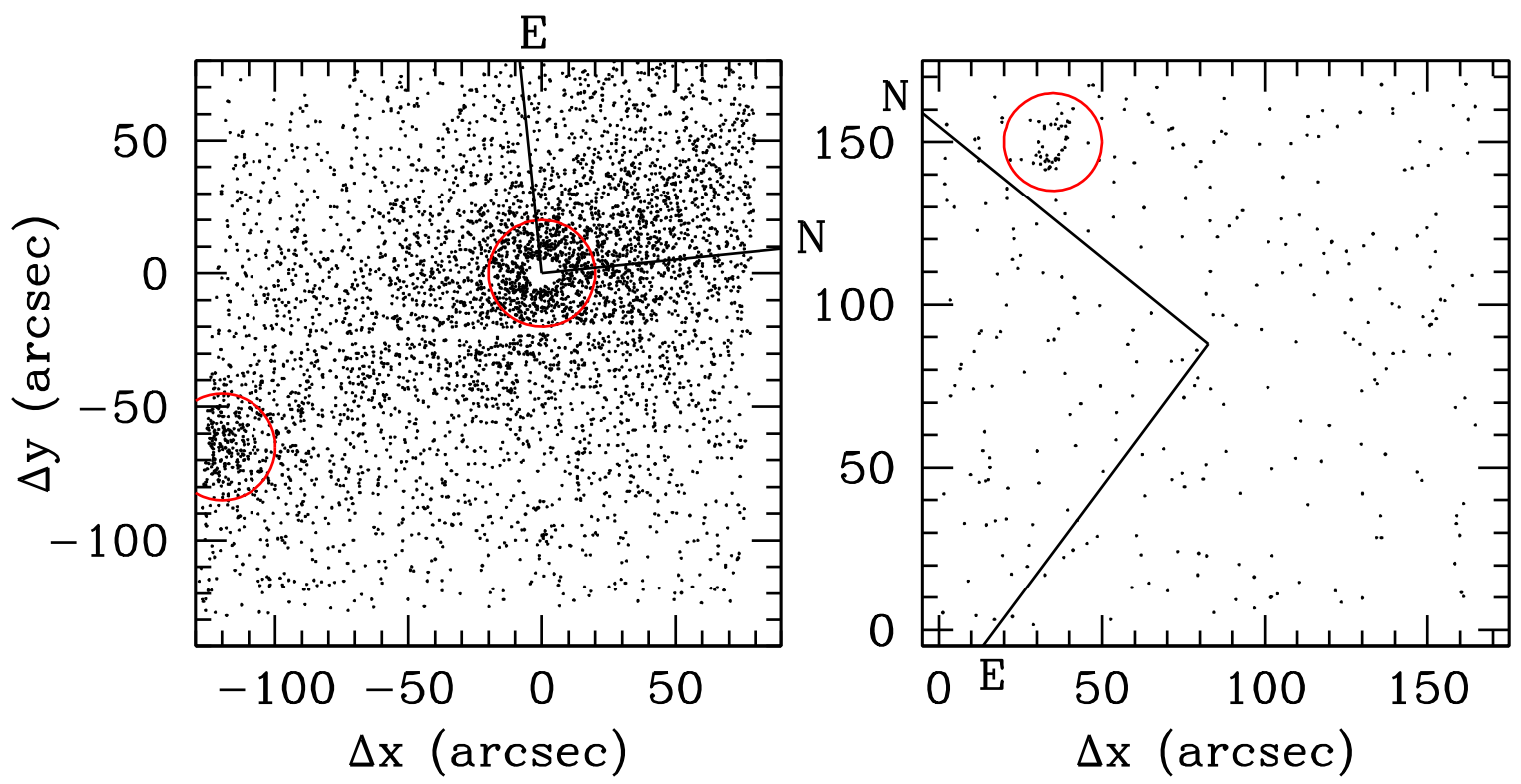

Figure 7. Locations of the measured starlike objects brighter than $F 814 \mathrm{~W}=27.0$ in the ACS field centered near UGC 10143 (left panel) and in the Parallel WFC3 field (right panel). In the ACS field the companion galaxy PGC056777 is shown by the $20^{\prime \prime}$ circle in the lower left, while in the WFC 3 field one small galaxy is marked by a $15^{\prime \prime}$ circle in the upper left (see the text).

marked by a $r=25^{\prime \prime}$ circle. In the upper right is the smaller elliptical PGC054530.

In Figure 7 we show the distribution of measured starlike objects with $F 814 W<27.0$ in the UGC 10143 fields. In the ACS field (left panel) one obvious clump of points at the lower left marks PGC056777 ( $r=20^{\prime \prime}$ circle), a nearly face-on disk galaxy with a complex and distorted array of spiral arms. In the WFC3 field, only one relatively small galaxy appears in the upper left $\left(r=15^{\prime \prime}\right.$ circle), which is 2MASXJ16023373 +1555259 .

The same photometric procedures were followed for NGC 4889 , with the resulting $x y$ plot as shown in Figure 8. The clump of points to the left of the central giant galaxy indicates a GC population around the nearby galaxy NGC 4886 (=NGC 4882), classified E0. In between these is the lenticular galaxy PGC044708, but this does not contribute significantly to the GC population.

As noted above, the GC population around NGC 4874 has been analyzed by Cho et al. (2016) from the single field NGC 4874-F2 as listed in Table 2. To add more statistical weight to our measurements of this rich GC system and especially to increase the radial coverage, we added data from two other overlapping fields with ACS exposures in the same filters (listed as NGC 4874-F1 and NGC 4874-F3 in Table 2), 


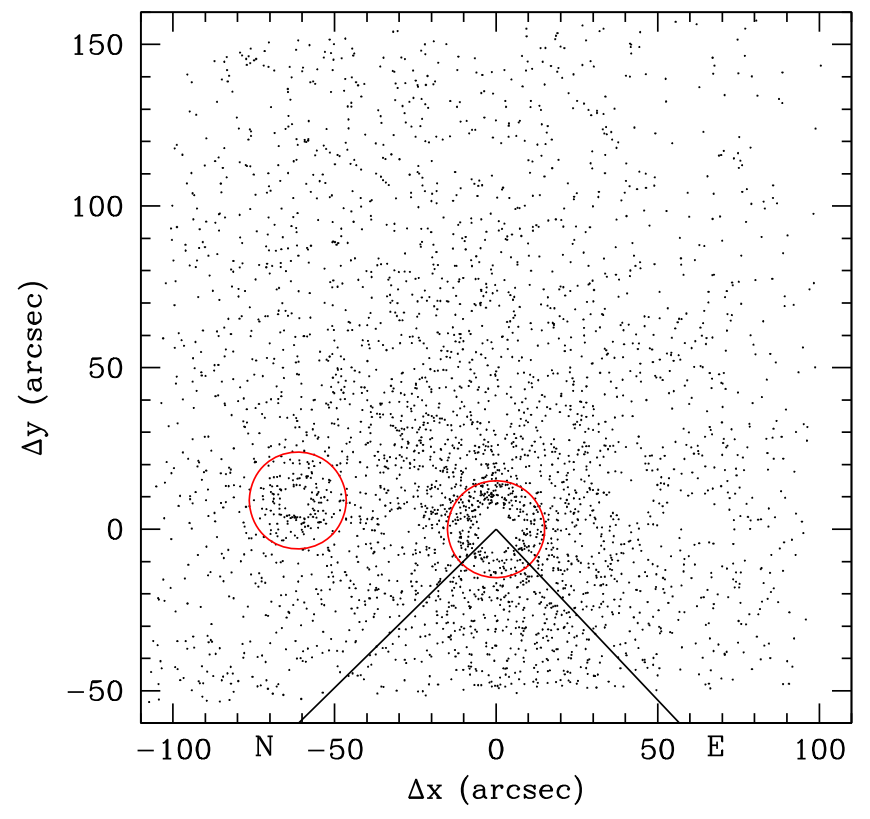

Figure 8. Locations of the measured starlike objects brighter than $F 814 W=26.5$ in the ACS field centered near NGC 4889. A circle of $20^{\prime \prime}$ radius is marked around both the center of NGC 4889 and the companion E0 galaxy NGC 4886 (at left).

although the exposures in field F2 reach the deepest of the three (see below). ${ }^{6}$ To guarantee that all three fields were on the same internal magnitude scale, we used the overlapping objects measured in more than one field to define mean magnitude offsets in both filters and normalize fields F1 and F3 to the magnitude scales of F2. These offsets were all smaller than \pm 0.03 mag in either filter, which is within the internal uncertainties of the large-aperture corrections to the allstar PSF-fitting magnitudes (see Paper II). To define a final photometric data set, we used F2, plus the regions of F1 and F3 that fall outside the area covered by F2. The $x y$ plot for the three fields combined is shown in Figure 9. As is evident in the figure, the camera orientation angles were different for each field, leaving a somewhat irregularly shaped composite field. The three smaller galaxies marked with $r=15^{\prime \prime}$ circles are NGC 4873 (at top), NGC 4875 (lower left), and NGC 4869 (lower right), and these are excluded from the later analysis.

The color-magnitude diagrams for UGC 9799 are shown in Figure 10. The pattern seen in the CMD is reminiscent of what we found for NGC 6166 (Paper II), with a noticeable blue MP sequence centered near $(F 475 W-F 814 W) \simeq 1.6$ and a broader distribution of objects to the red, but with no clear "valley" at intermediate colors. In the WFC3 field (unlike for NGC 6166), remarkably few objects are seen in the expected GC color range, suggesting that the "intragalactic" GC population within A2052 is small. We note, however, that the data plotted here comprise only the objects with $y<95^{\prime \prime}$ in Figure 6. We used only the lower part of the WFC3 field to avoid the contamination from the smaller galaxies in the top half of the field. For WFC3, the large number of objects fainter than the $50 \%$ completeness line is a result of a very

\footnotetext{
6 The measurements by Cho et al. are in the ABMAG system, whereas our independent reductions are in the VEGAMAG system to make them strictly comparable with the other BCGs in our study. Their photometry also uses SExtractor parameters for the photometry, whereas our data are from daophot/ allstar; we used SE only for object detection and preliminary culling.
}

conservatively faint initial detection threshold, which means that most of these "objects" are most likely not real. None of our analyses uses data fainter than the completeness limit.

For UGC 10143, the ACS and WFC3 CMDs are shown in Figure 11. The overall color distribution resembles UGC 9799, although a closer look shows relatively few red (MR) clusters; this is quantified in the discussion below. Again, very few objects in the GC color range appear in the WFC3 field, where we have excised only the data points within $15^{\prime \prime}$ of the small galaxy in the upper left in Figure 7.

For NGC 4889, the CMD is shown in Figure 12. In this case a more noticeable classic separation between the MP and MR subpopulations is visible, primarily because the MP sequence is narrower than for the other BCGs discussed here. Still, significant overlap is present.

For NGC 4874, the CMDs of the three ACS fields (F1, F2, F3 in Table 2) are shown individually in Figure 13. The F2 data are clearly the deepest and slightly more internally precise than the shorter exposures of F1 and F3. The CMD for the combined fields is shown in Figure 14. The net result leaves 16064 objects over all magnitudes, although in this combined graph there is no single well defined limiting magnitude. All the data are highly complete $(f>0.9)$ for $F 814 W \lesssim 26.0$, however.

The GC populations in these BCGs consist of many thousands of objects and completely dominate any field contamination. In Paper II we used photometry from a local control field, similar data from the Hubble Ultra-Deep Field, and a standard model for the expected population of Milky Way foreground stars to evaluate the field contamination. The numbers of contaminating objects within the magnitude and color range of interest here $(I \simeq 22-27,(g-I) \simeq 1.2-2.5)$ amount to less than a dozen starlike objects per ACS field and thus are negligible by comparison with the GC populations. In what follows, no corrections are made for field contamination.

\section{THE COLOR DISTRIBUTION FUNCTIONS}

\subsection{UGC 9799 and UGC 10143}

For UGC 9799, the CDF for 6630 objects brighter than $F 814 W=27.0$ in the color range $(F 475 W-F 814 W)=$ $1.2-2.5$ and $R>10^{\prime \prime}$ from the galaxy center is shown in Figure 15. Quite clearly, the raw histogram has a unimodal skewed shape. However, a bimodal-Gaussian model applied to the data returns an excellent match to the entire histogram: two modes are required, but more than two are unnecessary. As in Paper II, we use here the GMM fitting code (Muratov \& Gnedin 2010). In NGC 6166 we found the same pattern-two broad and heavily overlapped CDF modes with no minimum or "valley" between them-but UGC 9799 is even more extreme (compare Figure 11 from Paper II). Even though the mode peaks $\left(\mu_{1}, \mu_{2}\right)$ are separated by the same amount in color (0.32 mag, corresponding to $\simeq 0.8 \mathrm{dex}$ in metallicity), the dispersions of each mode are distinctly larger. The $D$-statistic, a useful measure of the separation between modes relative to their dispersions (see Muratov \& Gnedin and Paper II), is $D \simeq 1.70$, below the $D \gtrsim 2$ range where intrinsic bimodality can be strongly favored.

The fitting parameters for comparison are listed in Table 3. Here, the MP and MR modes have peak colors $\left(\mu_{1}, \mu_{2}\right)$ and dispersions $\left(\sigma_{1}, \sigma_{2}\right), p_{1}$ is the fraction of objects belonging to the MP mode, and $D$ measures the statistical significance of the 


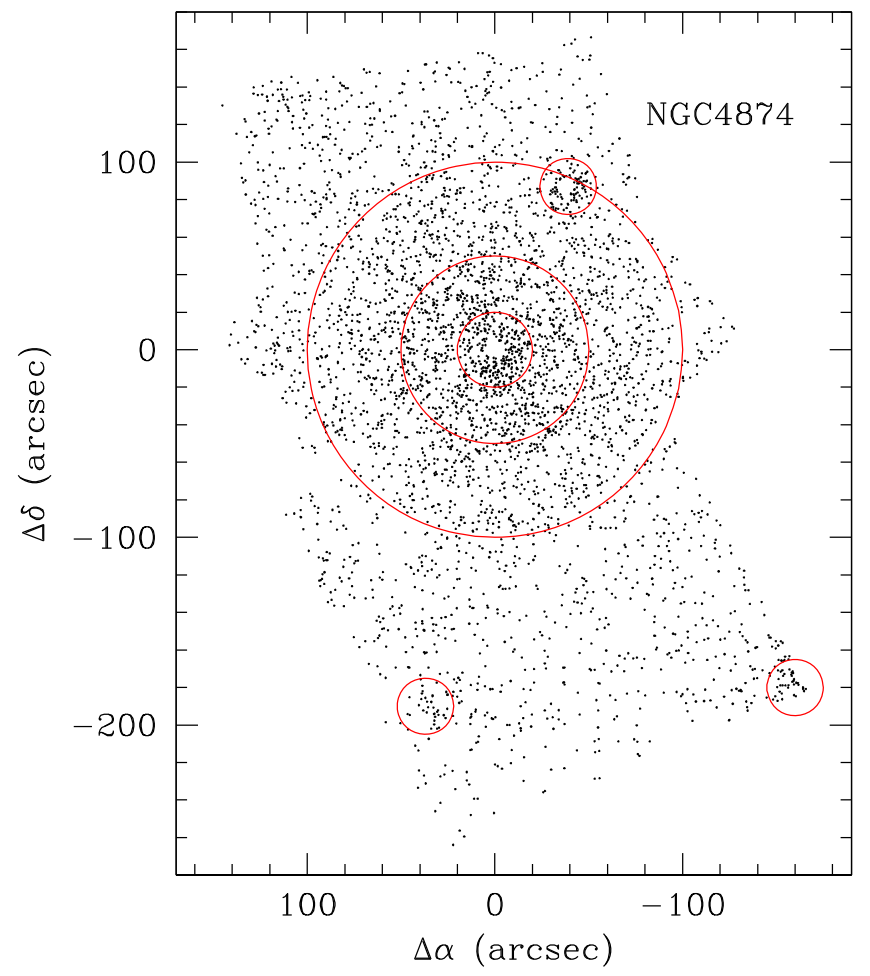

Figure 9. Locations of the measured starlike objects brighter than $F 814 W=26.0$ in the three overlapping ACS fields centered near NGC 4874. Coordinates plotted are relative to the center of NGC 4874, aligned following the cardinal axes with east at left and north at top. Three small galaxies with noticeable GC populations of their own are marked with red circles of $15^{\prime \prime}$ radius. The concentric circles around the center of NGC 4874 have radii of $20^{\prime \prime}, 50^{\prime \prime}$, and $100^{\prime \prime}$.
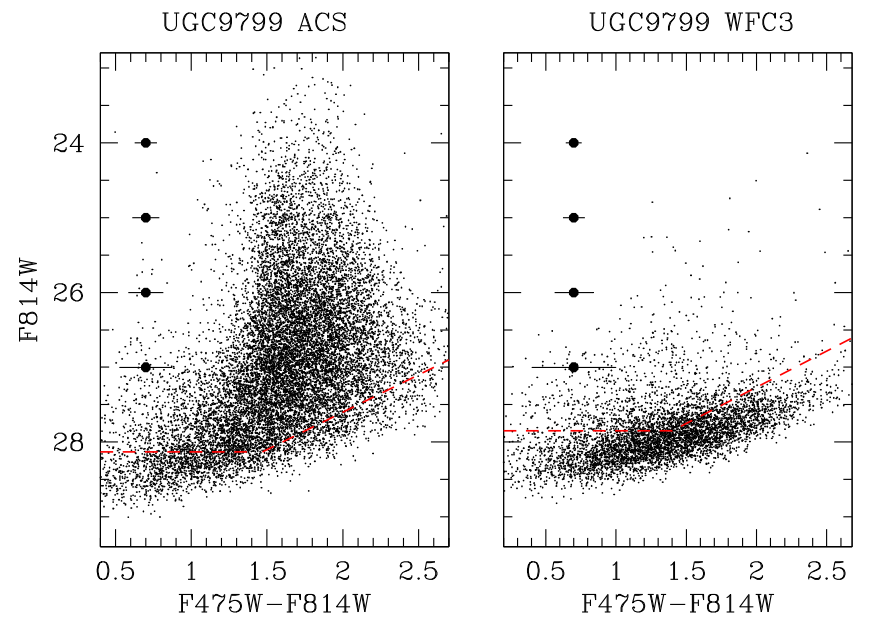

Figure 10. Color-magnitude diagrams for the measured starlike objects around UGC 9799, for the ACS/WFC field (left panel) and the outlying WFC3/WFC field (right panel). Here the native filter magnitudes $F 475 \mathrm{~W}, F 814 \mathrm{~W}$ are plotted, closely equivalent to $I$ vs. $\left(g^{\prime}-I\right)$ in the VEGAMAG system. These values are not corrected for reddening. For the WFC 3 field, objects with $y<95^{\prime \prime}$ are plotted to avoid contamination from another galaxy (see text). Detection completeness levels of $f=0.5$ are marked with the red dashed lines, and the photometric measurement uncertainties are indicated by the error bars at left.

mode separation. For UGC 9799, the ACS field excludes the two satellite galaxies marked in Figure 6, while the WFC3 field excludes the upper part of the frame containing the smaller galaxies as described above.
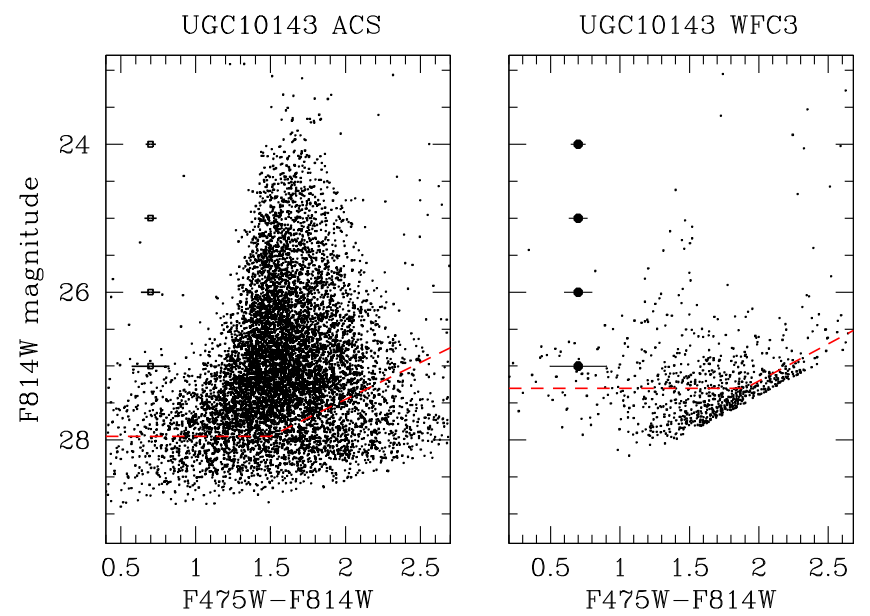

Figure 11. Color-magnitude diagrams for UGC 10143, for ACS (left) and WFC3 (right).

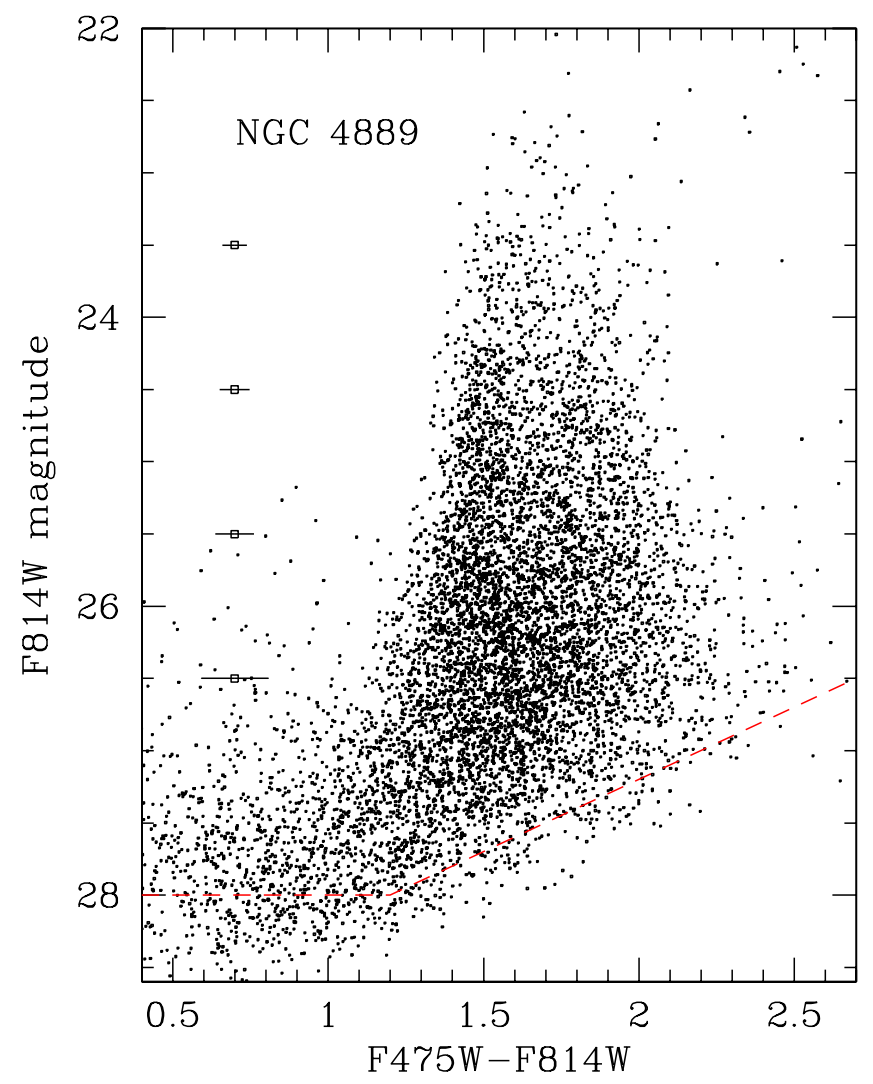

Figure 12. Color-magnitude diagram for 7892 measured starlike objects around NGC 4889 . Objects within $R<15^{\prime \prime}$ of either NGC 4889 or NGC 4886 are excluded.

For UGC 10143, the CDF for 3784 objects brighter than $F 814 W=27.0$ in the color range $(F 475 W-F 814 W)=$ $1.1-2.3$ and $R>20^{\prime \prime}$ from the galaxy center is shown in Figure 16. The satellite galaxy in the lower left in Figure 7 is excluded. The CDF has a unimodal skewed shape as for UGC 9799, but again a bimodal Gaussian model produces an excellent fit with the results given in Table 3. The main difference compared with UGC 9799 is a noticeably lower proportion of the MR component (just $40 \%$ of the total GC population, compared with $\sim 60 \%$ for NGC 6166 and UGC 9799). 
NGC 4874

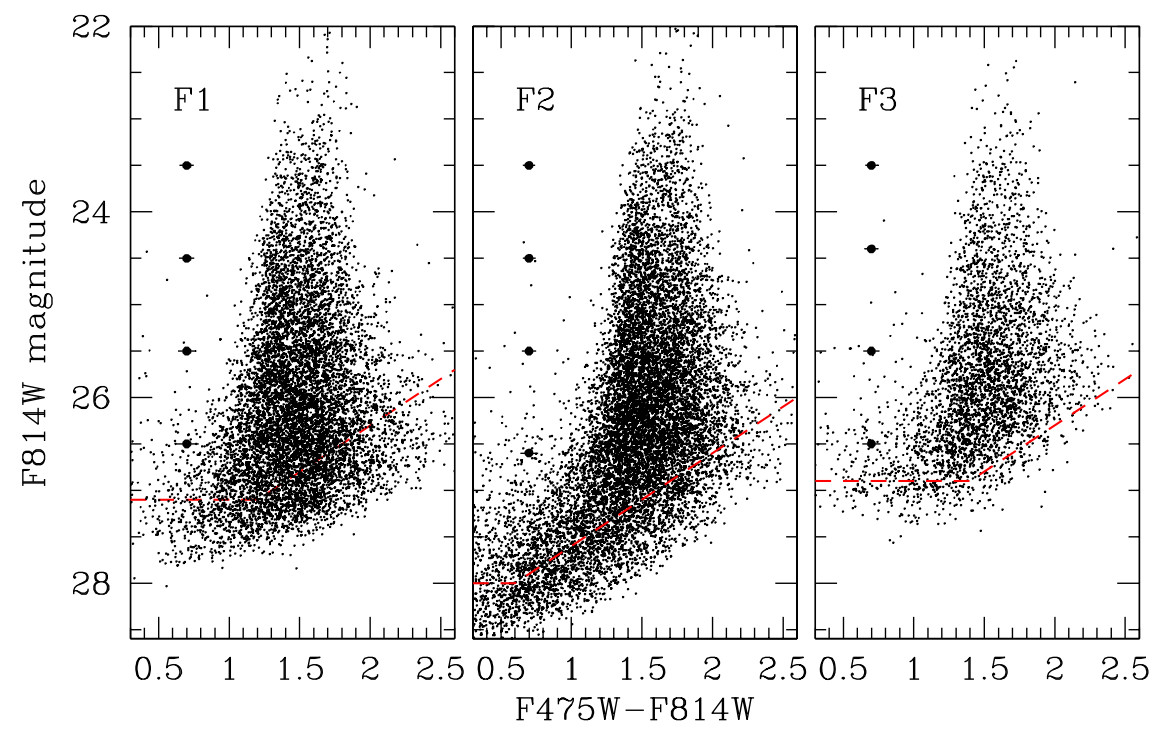

Figure 13. Color-magnitude diagrams for the measured starlike objects in the three fields F1, F2, and F3 around NGC 4874. F1 and F3 each overlap partially with F2, therefore the samples in these three diagrams are not fully independent. Note the differences in limiting magnitude.

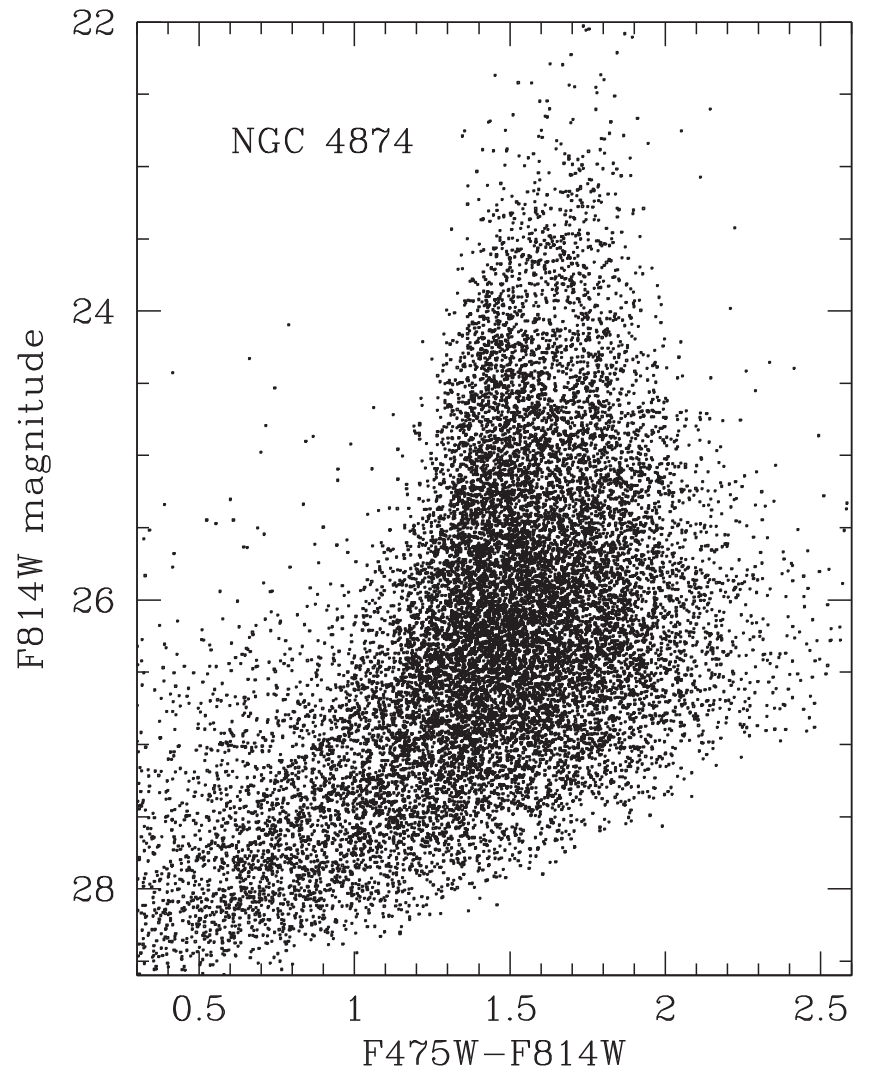

Figure 14. Color-magnitude diagram for the measured starlike objects around NGC 4874, for the combination of three fields as described in the text.

\subsection{The Coma Giants}

For NGC 4889, the CDF for 2956 objects in the range $F 814 W=23.5-26.0$ and $(F 475 W-F 814 W)=1.3-2.2$ is shown in Figure 17. The innermost circle $R<20^{\prime \prime}$, which is most affected by background light and incompleteness, is not included. The best-fit bimodal Gaussian shown in the figure reproduces the wings of the $\mathrm{CDF}$ and the blue peak well, but in the intermediate color range $\sim 1.6-1.8$, where noticeable

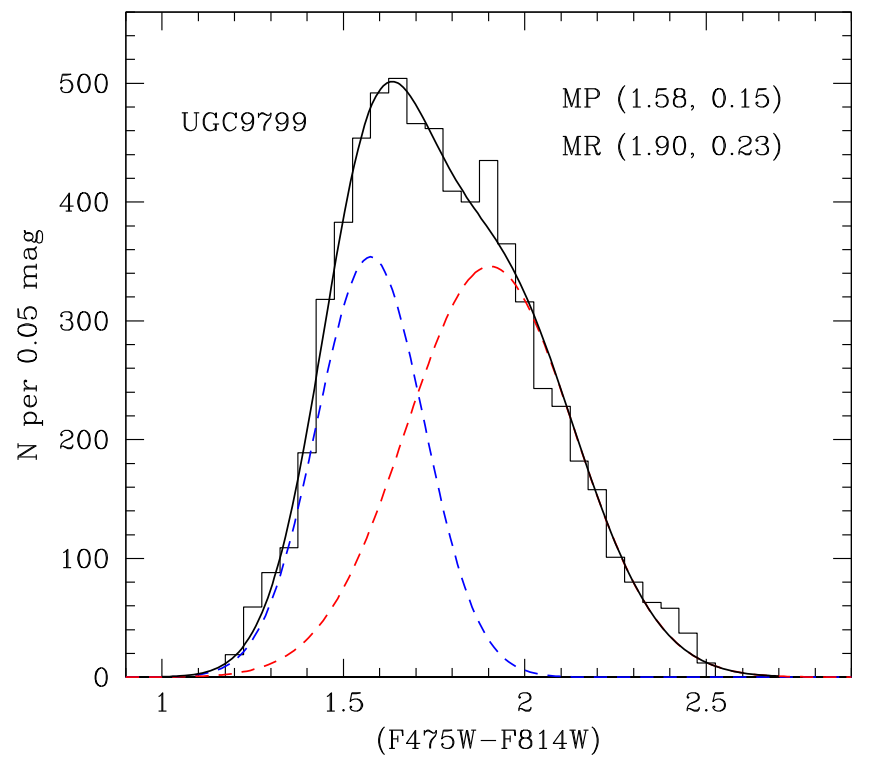

Figure 15. Color distribution function (CDF) for objects around UGC 9799 with $F 814 W<27.0$ and $R>10^{\prime \prime}$. A bimodal Gaussian fit to the data is shown by the superimposed curves; the numbers in the upper right give the means and standard deviations of the two modes.

discrepancies with the model occur, the performance is poorer. Attempts at adding a third or fourth mode do not improve the fit (the GMM solution damps these additional modes down to negligible levels).

Last, for NGC 4874, we show the CDF for 5140 objects in the magnitude range $F 814 W=24.0-26.0$ and color range $(F 475 W-F 814 W)=1.2-2.2$ in Figure 18 . We restrict the magnitude range at the bright end $(F 814 W<24)$ to deliberately avoid the high-luminosity end of the GC distribution where the CDF becomes unimodal (see Cho et al. 2016, and the next section). We also exclude the range fainter than $\simeq 26$, where the Field 3 data start to become incomplete. Fortunately, an adopted limit of $F 814 W \simeq 26.0-26.5$ for the Coma giants corresponds to about the same luminosity $\left(M_{I} \simeq-9\right)$ as in UGC $9799 / 10143$, which are about one magnitude more 
Table 3

Bimodal Gaussian Fits

\begin{tabular}{|c|c|c|c|c|c|c|c|c|}
\hline Galaxy & $F 814 W$ Range & $n$ & $\mu_{1}( \pm)$ & $\mu_{2}( \pm)$ & $\sigma_{1}( \pm)$ & $\sigma_{2}( \pm)$ & $p_{1}$ & $D$ \\
\hline NGC 6166-ACS & $23.0-26.5$ & 4712 & $1.401(0.009)$ & $1.719(0.015)$ & $0.122(0.005)$ & $0.178(0.006)$ & $0.42(0.04)$ & $2.08(0.12)$ \\
\hline NGC 6166-WFC3 & $23.0-26.5$ & 147 & $1.324(0.021)$ & $1.674(0.074)$ & $0.136(0.033)$ & $0.244(0.013)$ & $0.71(0.12)$ & $1.77(0.95)$ \\
\hline UGC 9799-ACS & $20.0-27.0$ & 6630 & $1.575(0.009)$ & $1.904(0.013)$ & $0.148(0.005)$ & $0.231(0.002)$ & $0.40(0.03)$ & $1.70(0.03)$ \\
\hline UGC 10143-ACS & $24.0-27.0$ & 3784 & $1.516(0.011)$ & $1.818(0.033)$ & $0.138(0.006)$ & $0.193(0.012)$ & $0.60(0.07)$ & $1.80(0.19)$ \\
\hline UGC 10143-WFC3 & $24.0-27.0$ & 119 & $1.473(0.093)$ & $1.921(0.092)$ & $0.205(0.048)$ & $0.083(0.048)$ & $0.77(0.20)$ & $2.87(0.31)$ \\
\hline NGC 4889 & $23.5-26.0$ & 2956 & $1.497(0.006)$ & $1.802(0.009)$ & $0.084(0.003)$ & $0.163(0.004)$ & $0.40(0.02)$ & $2.35(0.09)$ \\
\hline
\end{tabular}

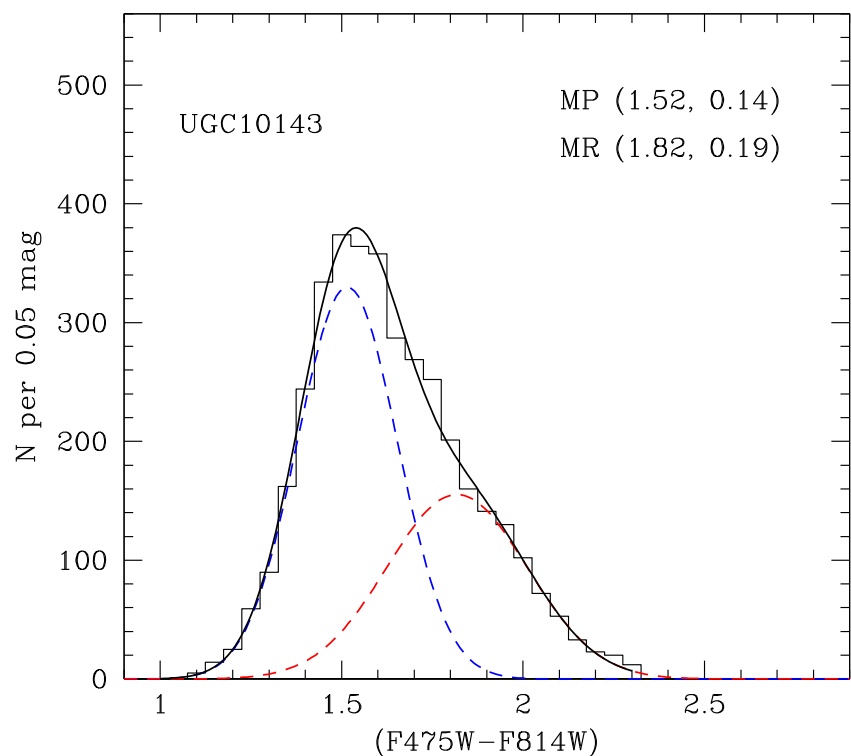

Figure 16. CDF for objects around UGC 10143 with $F 814 W<27.0$ and $R>20^{\prime \prime}$. A bimodal Gaussian fit and its parameters are shown by the superimposed curves and the numbers in the upper right, as in the previous figure.

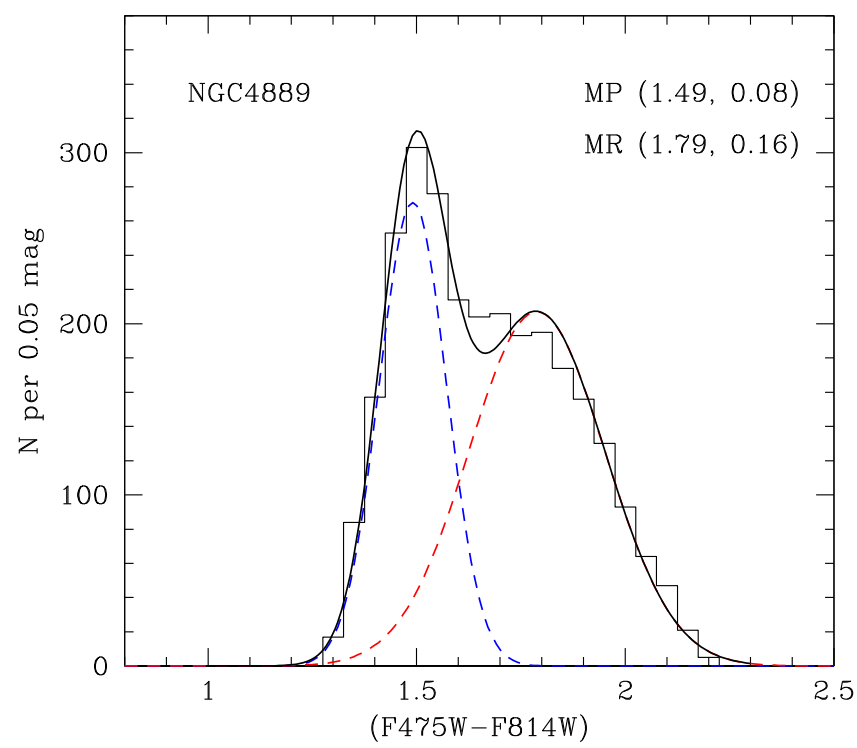

Figure 17. CDF for objects around NGC 4889 with $F 814 W=23.5-26.0$ and $R>15^{\prime \prime}$. A bimodal Gaussian fit and its parameters are shown by the superimposed curves and the numbers in the upper right, as in the previous figure.

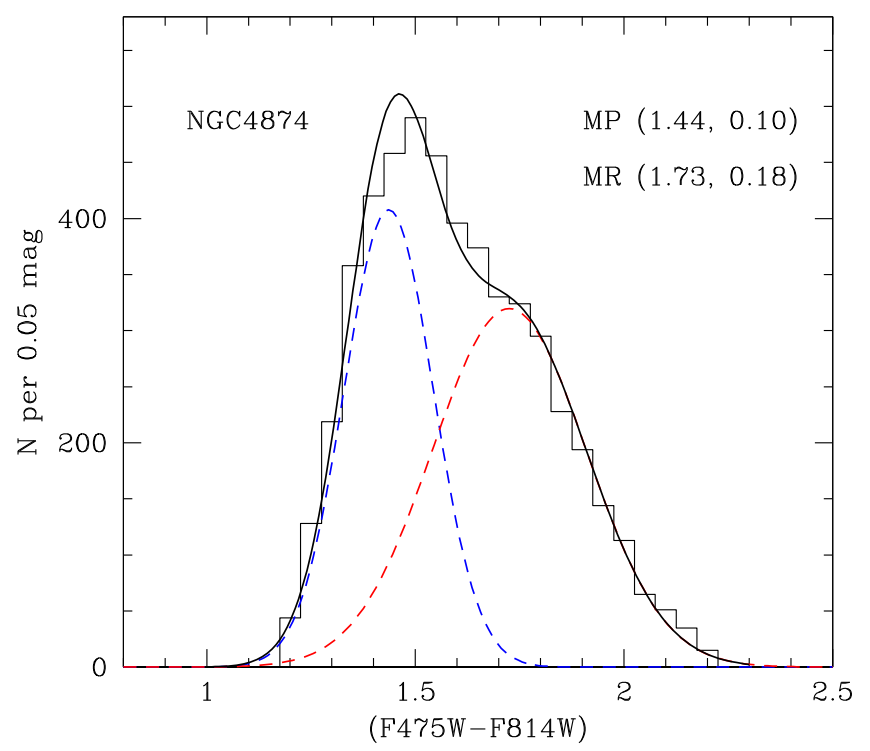

Figure 18. CDF for objects around NGC 4874 with $F 814 W=24.0-26.0$ and $R>20^{\prime \prime}$, along with the best-fit bimodal Gaussian.

distant. For NGC 4874, a bimodal Gaussian fit produces a match to the CDF, but again not as cleanly as in NGC 6166, UGC 9799, or UGC 10143: the color range near the blue peak stands out as discrepant. Nevertheless, a standard Kolmogorov test (i.e., a one-sample KS test) for the two Coma giants does not suggest a statistically significant deviation from the bimodal model. Just as for NGC 4889 above, adding more modes to the solution does not improve the overall fit, nor does the imposition of equal variances (homoscedasticity).

As we extensively discussed in Paper II, the internal precision of the color indices in the magnitude range of interest $(F 814 W \lesssim 27)$ is high enough to be easily capable of resolving the widths $\left(\sigma_{1}, \sigma_{2}\right)$ of the two modes without adding significant spreading to either one. The evidence then suggests that these high color dispersions seen in all of the BCGs, along with the near-linearity of the $(g-I)$ color index, are due to the intrinsic metallicity spread of each mode.

\subsection{MP and MR Populations Versus Radius: Gradients?}

Previous evidence for many large galaxies (Paper II and references cited above) shows the common existence of radial metallicity gradients in their GC systems, which are primarily detected as a changing ratio of blue to red clusters with galactocentric radius. A convenient way to characterize these gradients is to plot the blue fraction $p_{1} \equiv N($ blue $) / N($ total $)$ 


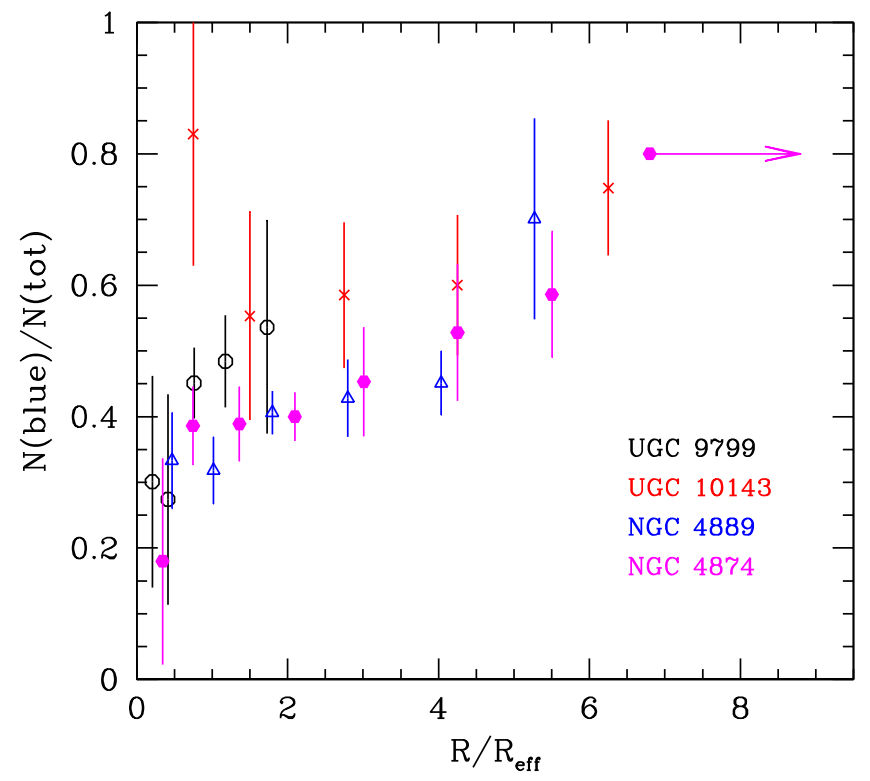

Figure 19. Blue fraction $p_{1}=N($ blue $) / N$ (tot) as a function of projected galactocentric distance. The outermost point for NGC 4874 (magenta arrow in the upper right) refers to the GCs in the intragalactic medium of the Coma cluster.

versus $R_{\mathrm{gc}}$. For the four galaxies discussed here, the data for $p_{1}(R)$ are shown in Figure 19. In each case, GMM fits were made in radial zones, and the mean $\langle R\rangle$ for the clusters in each zone is expressed as a ratio of the effective radius $R_{\text {eff }}$ of the galaxy light profile.

Although noticeable differences occur between galaxies in the overall mean level of $p_{1}$, a repeated trend emerges for $p_{1}$ to rise fairly steeply from $R=0$ out to $\simeq 1.5 R_{\text {eff }}$, then to plateau or rise more gradually until the blue fraction reaches $p_{1} \sim 0.5$, and finally beyond $\sim 4 R_{\text {eff }}$ to increase more steeply again. For NGC 4874, we have added an outermost data point from Peng et al. (2011), who note that the intragalactic GC population becomes progressively more dominant beyond $R \simeq 270^{\prime \prime}$ ( $7 R_{\text {eff }}$ ) and that the ICL has $p_{1} \simeq 0.8$. The ICL value is shown in the figure as an outward arrow.

As emphasized above, a major result of this study is that the MDFs summed over all radii are broad with heavy overlap between the standard MP and MR sequences. Two straightforward ways in which this result could be generated are that (a) the intrinsic dispersions of the two modes are actually small at a given radius, but there is a strong radial gradient in the mean colors $\mu_{1}, \mu_{2}$ so that the two modes are blurred when all radii are combined; or alternatively, that (b) the intrinsic dispersions $\sigma_{1}, \sigma_{2}$ are high at all galactocentric radii, so that any smaller radial zone would be fairly representative of the whole. In option (a), the MDF should appear more distinctly bimodal the more restricted the range of radii.

Option (b) appears to be the correct one. To test option (a) directly, we performed GMM bimodal-fit solutions in smaller radial bins. These show that there is no significant variation of either $\mu_{1}$ or $\mu_{2}$ with radius (see also Paper II for NGC 6166 in more detail). A direct test of option (b) is presented in Figure 21, where we show the dispersions $\sigma_{1}, \sigma_{2}$ versus mean radius, and where $R$ is renormalized to the same physical scale (kiloparsecs) for all five galaxies discussed here. There are some differences in the mean $\sigma$ s from one galaxy to another, but the clear signal from this plot is that the two modes are both quite broad at all radii, and furthermore, that within any one GCS they undergo very little change with $R$. In other words, the MP and MR modes heavily overlap at all projected locations in their halos. As expected, the most obviously bimodal system of the five (NGC 4889) has the lowest $\sigma$-values in both modes. (We recognize here, again, that for cases such as UGC 9799 and UGC 10143, where the MDFs are in fact quite smooth and continuous, discussing them in terms of two clearly distinguishable "modes" begins to look like only a numerical exercise).

The combined evidence points to a picture where the mean radial metallicity gradient in the entire GCS is entirely due to a population gradient, which is observed as a steadily increasing ratio of MP to MR clusters with increasing radius. Very similar results-little change with radius in the mean colors and dispersions of both modes, combined with a radial increase in $f($ blue $)$ - have been found for other giant ellipticals, including M87, M49, and M60 (Geisler et al. 1996; Lee et al. 2008; Harris 2009b), NGC 1399 (Bassino et al. 2006), NGC 6861 (Escudero et al. 2015), NGC 4278 (Usher et al. 2013), and other BCGs and giants (Bassino et al. 2008; Harris 2009a; Faifer et al. 2011). One notable exception is NGC 1407, which shows distinct radial decreases in mean color (Forbes et al. 2011); small but nonzero gradients appear in intermediateluminosity Fornax members (Liu et al. 2011).

\subsection{Mass-Metallicity Trends}

In NGC 6166 we found clear evidence of a mass-metallicity relation (MMR) along the blue sequence in the sense that mean GC color becomes redder at higher luminosity. Quantitatively, the effect corresponded to a simple power law where heavyelement abundance scales with GC mass as $Z \sim M^{\gamma}$, where $\gamma=0.27 \pm 0.06$ over almost the entire luminosity range brighter than the GCLF turnover point. A scaling similar to this along the blue sequence has been found in several other giant and supergiant ellipticals at high statistical significance (e.g., Harris et al. 2006; Mieske et al. 2006; Strader et al. 2006; Wehner et al. 2008; Cockcroft et al. 2009; Peng et al. 2009; Faifer et al. 2011; Fensch et al. 2014; Cho et al. 2016, among others). No galaxy has revealed convincing nonzero color trends along the red MR sequence.

For UGC 9799 and UGC 10143, the results from a similar analysis are shown in Figure 20. For these two galaxies, the strong overlap between the MP and MR components essentially continues more or less unchanged upward to the brightest magnitude ranges, making clear conclusions about color slopes quite difficult. For UGC 9799, we quantitatively find for the blue sequences $\gamma=0.07 \pm 0.12$ when we use only the points in the range $24.5<F 814 W<27.0$, but if we use all points $F 814 W<27.5$, then $\gamma=0.48 \pm 0.21$. For this galaxy, we cannot rule out any of $\gamma \sim 0$, a positive slope similar to other cases, or a nonlinear solution. For UGC 10143, the mean points along the MP sequence give a much more consistent value $\gamma=0.01 \pm 0.05$, i.e., indistinguishable from zero.

In NGC 4889, which has more clearly separated blue and red modes, a better defined trend along the blue sequence is seen, giving for a linear fit $\gamma=0.25 \pm 0.06$. This is very similar to the result we found for NGC 6166, $\gamma=0.27 \pm 0.06$.

In NGC 4874, Cho et al. (2016) find strong evidence for a blue-sequence MMR, and we do not repeat their extensive analysis here. They find a nonlinear trend that becomes steeper at brighter magnitudes, which is thus not well described by a 


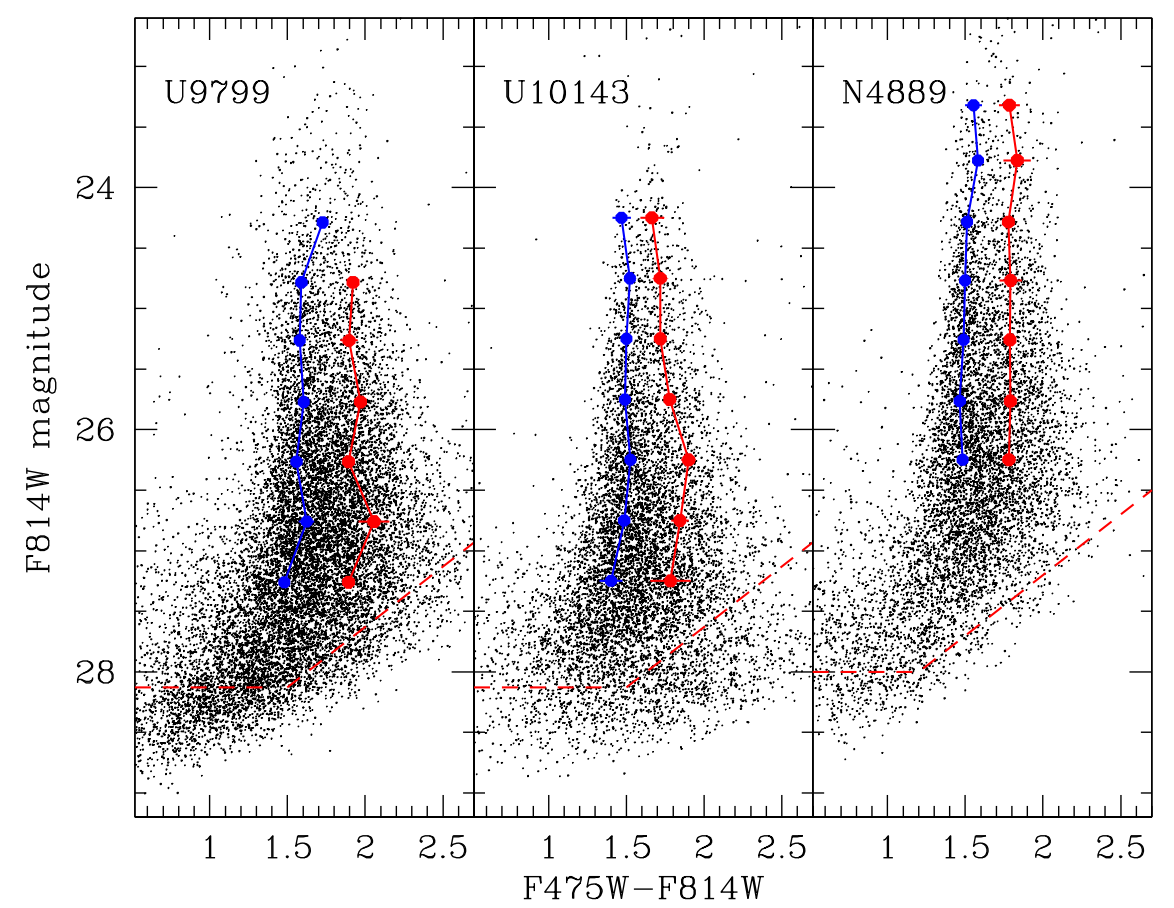

Figure 20. Mean points along the blue (MP) and red (MR) sequences for UGC 9799, UGC 10143, and NGC 4889, calculated in 0.5 mag intervals. In these three galaxies, little or no evidence appears of a systematic trend of GC color with luminosity.

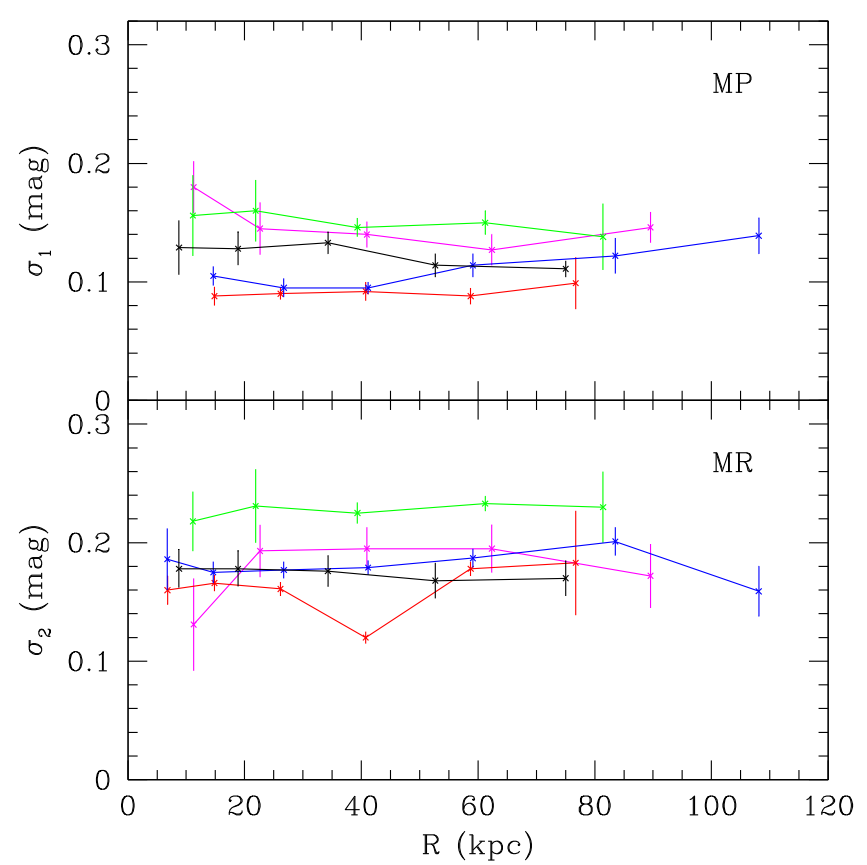

Figure 21. Upper panel: Gaussian width $\sigma_{1}$ of the blue (MP) sequence for the five BCGs discussed in this paper, plotted as a function of radius, in kiloparsecs. Lower panel: width of the red (MR) sequence $\sigma_{2}$ vs. $R$. NGC 6166 is plotted in black, NGC 4874 in blue, NGC 4889 in red, UGC 9799 in green, and UGC 10143 in magenta.

single slope $\gamma=$ const. Nevertheless, from our data using field F2 alone (the deepest and most internally precise part of the photometry), we find $\gamma=0.26 \pm 0.05$ using only a simple linear fit for $F 814 W<26$. For $F 814 W \lesssim 24$, the $\mathrm{CDF}$ becomes much more nearly unimodal and symmetric; in this high-luminosity range, blue GCs become very rare while the red sequence continues upward.

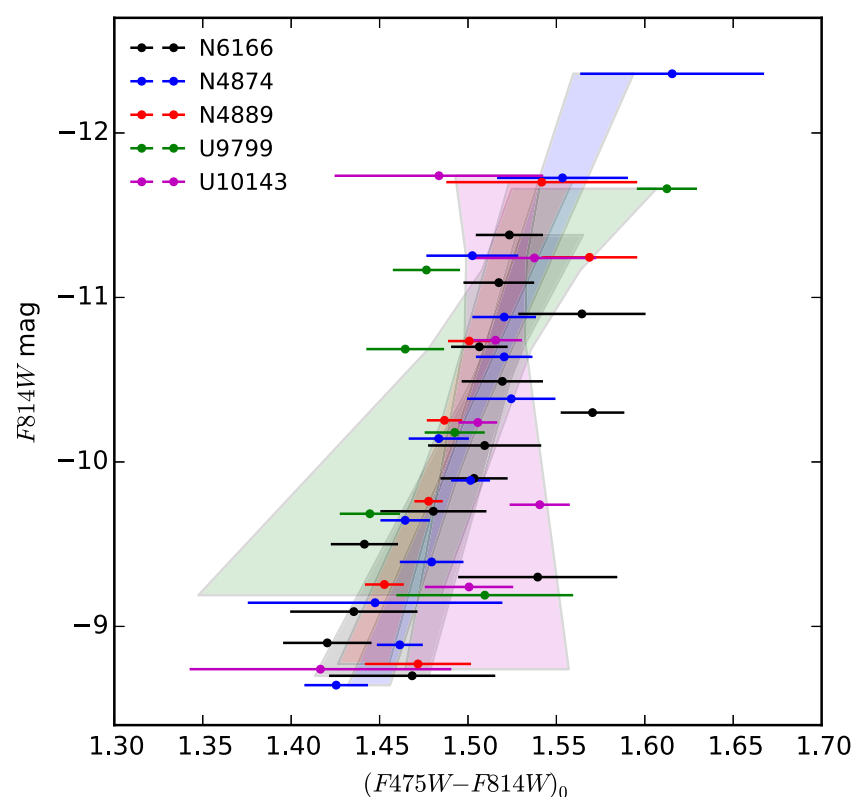

Figure 22. Mass-metallicity relations for the blue (metal-poor) sequences in the five galaxies discussed here. For each galaxy the shaded region shows the $\pm 1 \sigma$ uncertainty of the slope and intercept. NGC 6166 is plotted in gray, NGC 4874 in blue, NGC 4889 in red, UGC 9799 in green, and UGC 10143 in magenta. Note the result for UGC 9799 (light green region) is for a particular selection of the data points; see text.

A composite graph for the blue sequences in all five BCGs discussed here is shown in Figure 22. Here, the sequences have been shifted to a mean color $(g-I)_{0}=1.5$ for all of them, to enable better direct comparison. UGC 9799 is shown with the solution from all the mean points, giving a positive but very uncertain slope. NGC 4874, 4889, and 6166 are mutually very consistent at $\gamma \sim 0.25$, while UGC 10143 fairly clearly shows no color trend. 
As more BCGs are added to the analysis, it is becoming clearer that the existence of an MMR (or lack of one) is not universal, and even where one is present, no single description (such as $\gamma=$ const) may necessarily be valid. UGC 9799 and UGC 10143 in Figure 20 can be added to NGC 4472 in Virgo, and NGC 1399 in Fornax, as supergiant ellipticals that do not exhibit a definite MMR. The model most often adopted to produce the blue-sequence trend is that of Bailin \& Harris (2009), which is based on the approach that self-enrichment of the cluster stars takes place while the GC is still in its formation stages and thus while both gas and young stars are mixed together. Quantitative examples of fits of this self-enrichment model to various data are reported in Mieske et al. (2010), Cockcroft et al. (2009), Forbes et al. (2010), Harris et al. (2010), and Fensch et al. (2014). It is worth emphasizing that the use of BCGs in particular for model tests is crucial, because only the BCGs have large enough numbers of GCs to populate the highest-luminosity range $L \gtrsim 10^{6} L_{\odot}$ where the color trend becomes most obvious.

In Paper II we suggested that the Bailin/Harris model, at least in its basic form, has difficulty matching the observed range of MMRs (essentially, the observed range of bluesequence slopes $\gamma$ ). If the MMR is entirely due to selfenrichment, then it should be driven by very local conditions for the structure of the proto-GC, primarily its mass and central concentration (scale radius). The higher the mass and the smaller the scale radius, the more efficiently gas is retained in the protocluster and the more effect pre-enrichment can have. This scenario then suggests that the MMR should look similar from one galaxy to the next, however, with no immediately obvious role for larger-scale environmental effects that could differ strongly between host galaxies. In addition, selfenrichment in this model is highly nonlinear in cluster mass, and thus it would predict that the slope $\gamma$ should increase with GC mass, which is not observed in all cases. Another and more physically based obstacle faced by internal self-enrichment is simply that it would require a fairly extended initial star formation period of $\gtrsim 10 \mathrm{My}$ for the first round of SNe II to appear and contaminate the remaining gas in the protocluster, which could then proceed to form more low-mass stars of higher metallicity (see Bailin \& Harris 2009). Direct modeling of GC formation within giant molecular clouds indicates, however, that most of the star formation occurs within $\lesssim 4$ My (e.g., Hartmann et al. 2012; Howard et al. 2016). Observations of young clusters indicate small internal age ranges as well (e.g., Lada \& Lada 2003; Melena et al. 2008; Andersen et al. 2009; Schneider et al. 2014). The high-mass regime suitable for GCs has, however, not yet been probed either in theory or observation: ideally, we would like to determine the expected internal age range for young clusters at masses well above $10^{5} \mathrm{M}_{\odot}$. It is potentially promising that in the recent $\mathrm{Li}$ et al. (2016) models, cluster formation proceeds over $\gtrsim 15 \mathrm{My}$ in some of the most massive cases.

An alternative approach, although still simplistic at this point, would be to introduce pre-enrichment of the proto-GCs (VanDalfsen \& Harris 2004; Forte et al. 2007) and invoke higher pre-enrichment for higher-mass clusters. Different amounts of pre-enrichment among GCs, presumably drawn from their host giant molecular clouds, would in principle allow for a wider range of environmental influences. It is not yet clear, however, exactly how pre-enrichment should depend on GC mass.

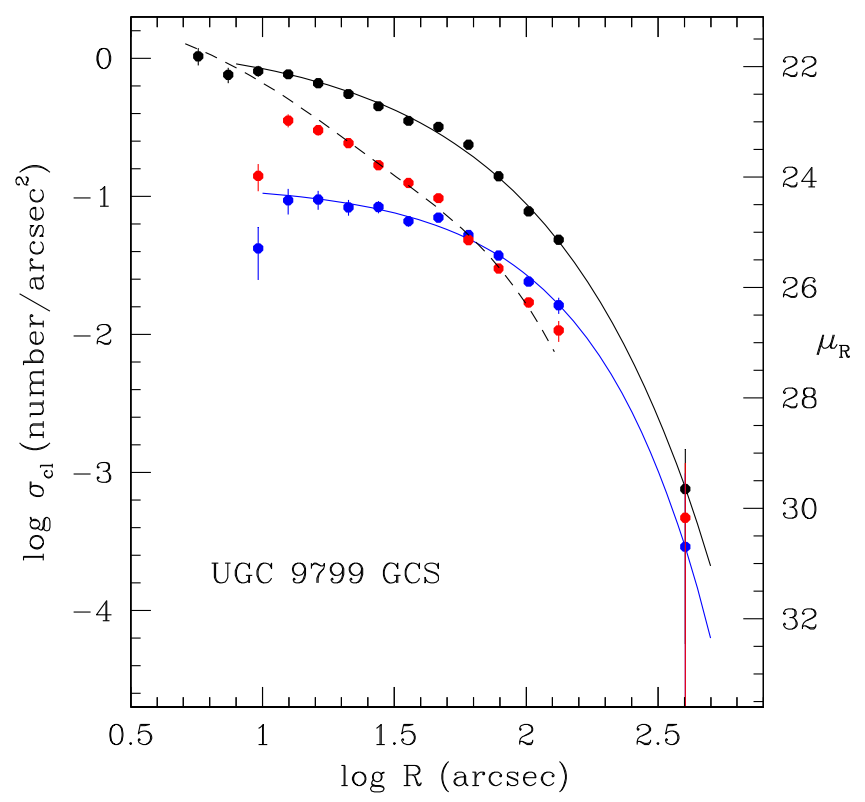

Figure 23. UGC 9799: radial profile for the entire GC system (black points and the solid curve), the EMP clusters (blue points and curve), and the EMR clusters (red points and curve). The dashed line fitted to the EMR data points shows the integrated surface brightness profile of the galaxy's halo light in $\mu_{R}$ (Seigar et al. 2007) with the scale shown along the right-hand axis (see text).

BCGs are likely to be products of mergers, and the sheer number of mergers could also have differed noticeably from one case to another. If we then assume that their progenitors experienced different levels of pre-enrichment before the formation of their MP clusters, then in general the MP sequence in the final combined BCG should have a higher internal color dispersion and a weaker net MMR slope in the cases where more mergers took place. In observational terms, $\sigma_{1}$ should increase as $\gamma$ decreases. Some hints that this is the case can be seen from the comparison of UGC 9799 and UGC 10143 with the Coma giants, from the numbers in Table 3.

It also remains unclear whether the MMR phenomenon is connected with the multiple stellar populations that have been detected within massive GCs in the Milky Way, an issue that continues to be a serious challenge for modeling (e.g., Renzini et al. 2015). For additional discussion, see Paper II and Fensch et al. (2014).

\section{SPATIAL DISTRIBUTIONS}

\subsection{Radial Profiles}

At the distance of these target galaxies, the ACS field of view is large enough to enclose a significant radial range of their halos. In Figures 23-25 the projected radial distributions $\sigma_{\mathrm{cl}}$ (number of GCs per $\operatorname{arcsec}^{2}$ ) of the GCs are shown for UGC 9799, UGC 10143, and NGC 4889, along with fits to Sérsictype functions obtained by $\chi^{2}$-minimization,

$$
\sigma_{\mathrm{cl}}=\sigma_{e} \exp \left(-b_{n}\left[\left(\frac{R}{R_{e}}\right)^{1 / n}-1\right]\right)
$$

or else to simple power-law form where appropriate. In the first two figures, the outermost data points are from the outlying WFC3 field, which allow us to track $\sigma_{\mathrm{cl}}$ out to nearly $R \simeq 300 \mathrm{kpc}$. We note that the radial distributions for the GCs in NGC 4874 are analyzed by Peng et al. (2011) and Cho 


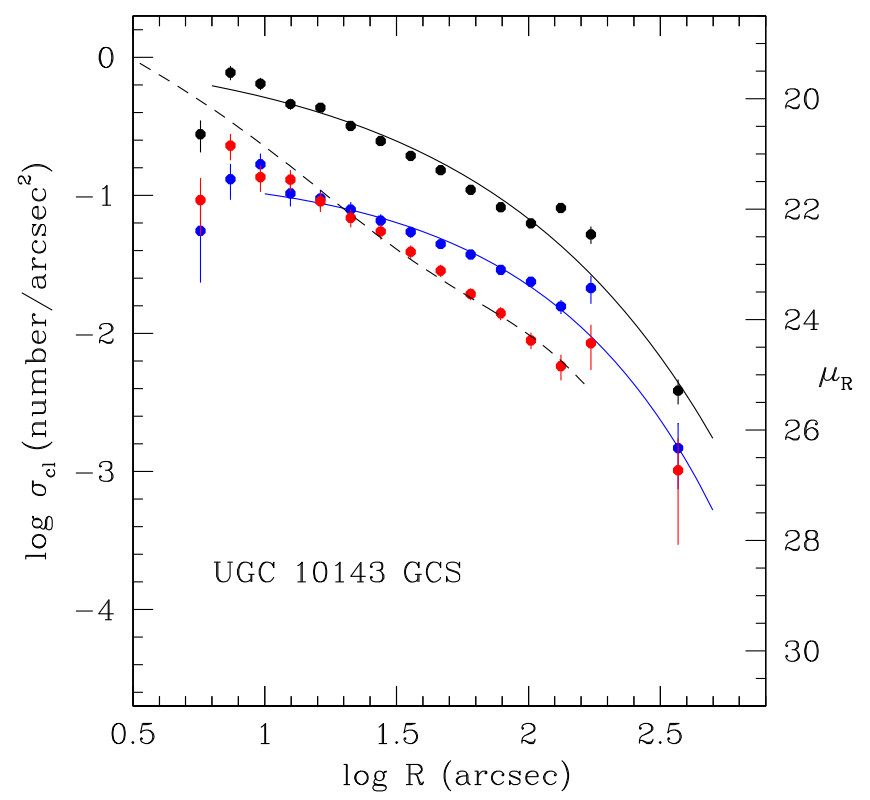

Figure 24. UGC 10143: radial profile for the entire GC system, the EMP clusters, and the EMR clusters, with symbols as in the previous figure. The Sérsic function fit is shown for the entire system (the black line) and the EMP clusters (the blue line). The dashed line fitted to the EMR data points shows the integrated surface brightness profile $\mu_{R}$ of the galaxy's halo light, from Donzelli et al. (2011).

et al. (2016), and we do not repeat their discussion here. They unambiguously find that the MP clusters follow a distinctly shallower distribution than the MR clusters.

The fact that the blue/red GC fraction increases with radius also means the radial profiles are a function of metallicity. To minimize the effects of the strong overlap between the MP and MR modes, we follow Paper II and define the extreme metalpoor (EMP) clusters as those bluer than the blue-mode peak, and extreme metal-rich (EMR) clusters as those redder than the red-mode peak. Although this step eliminates half the total GC sample, it gives a clearer view of the structural differences versus metallicity. In Figures 26-29 the $x y$ distributions for the EMP and EMR clusters in each galaxy are shown in the form of smoothed isocontour maps. The differences in central concentration are evident, with the EMP component distributed much more widely and often less symmetrically than the EMR clusters.

UGC 9799: The profile fits use the data $F 814 W<$ $27.0, R>10^{\prime \prime}$. The solutions for the radial fits are summarized in Table 4. The EMR component does not fit a single Sérsic profile as well, but it is certainly more centrally concentrated than the EMP component: very roughly, in simple power-law terms, for $R \gtrsim 30^{\prime \prime}$, the EMP clusters follow $\sigma \sim R^{-1.4}$ and the EMR clusters $\sigma \sim R^{-2.1}$. For comparison, in NGC 6166 we found $\sigma(\mathrm{EMP}) \sim R^{-1.0}, \sigma(\mathrm{EMR}) \sim R^{-1.8}$. Thus the overall halo of UGC 9799 is slightly more centrally concentrated, but the difference between EMP and EMR components is similar. Perhaps more importantly, the EMR distribution matches the surface brightness of the halo light well, as shown by the dashed line in Figure 23. Surface-brightness profiles in the $R$ filter have been measured by Seigar et al. (2007) with a double Sérsic profile out to $R \simeq 150^{\prime \prime}$, whereas Donzelli et al. (2011) measure it out to

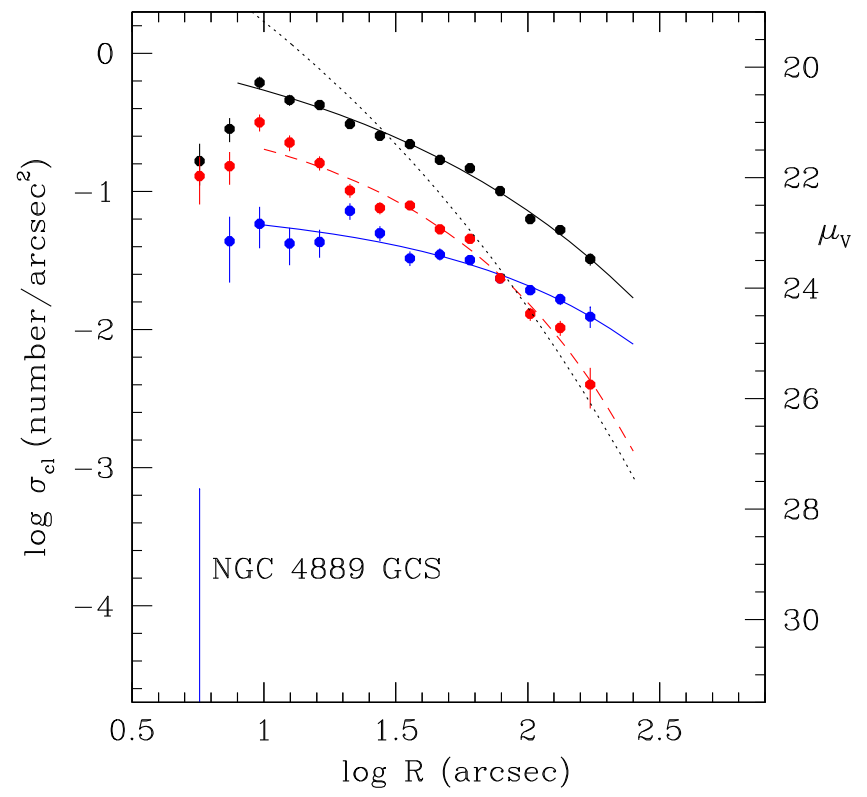

Figure 25. NGC 4889: radial profile for the entire GC system, the EMP clusters, and the EMR clusters, with symbols as in the previous figure. The Sérsic fits to each of the three components are shown as the solid line (all GCs), dashed blue line (EMP), and red line (EMR). The dotted line indicates the integrated $V$-band surface brightness profile $\mu_{V}$ of the halo light from Pahre (1999).

$R \simeq 100^{\prime \prime}$ and fit it to a single Sérsic profile. Here we adopt the data from Seigar et al. (2007).

UGC 10143: Again, we use all data $F 814 W<27.0, R>$ $10^{\prime \prime}$ for profile fitting. The EMR data more closely resemble a power law with $\sigma \sim R^{-1.2}$, only slightly steeper than the whole population. In this galaxy, both GC components therefore appear to follow rather shallow distributions, although at large radii the EMP component strongly dominates in total numbers. For the halo light profile, Donzelli et al. (2011) fit a twocomponent model with an inner Sérsic and outer exponential profile; the exponential part contains almost $80 \%$ of the total luminosity. In the same way as for UGC 9799 and NGC 6166, there is a close match between the EMR cluster distribution and the halo light.

NGC 4889: In this case, we use data in the range $F 814 W<26.5,1.3<(g-I)<2.2$. Before carrying out radial fits to a Sérsic function, we attempted to assess the contribution to the GC population in the field from the companion galaxy NGC 4886, which is a moderately large elliptical around which a noticeable GC system is seen (Figure 8). A numerical approach similar to that described in Wehner et al. (2008) for the Hydra BCG NGC 3311 and its companion NGC 3309 was used here: the field is divided into a grid of small $10^{\prime \prime} \times 10^{\prime \prime}$ squares, and the observed number of GCs within each square is assumed to be the sum of the contributions from both galaxies combined. With the assumption that their GC systems follow Hubble-type or Sérsic profiles, a $\chi^{2}$-minimization can then be used to solve for the profile parameters (see Wehner et al. for details). NGC 4886 was found to contribute negligibly to the totals beyond a $15^{\prime \prime}$ circle centered on it, therefore we exclude that region of the image and fit a single Sérsic profile centered on NGC 4889 alone using the remaining area. 

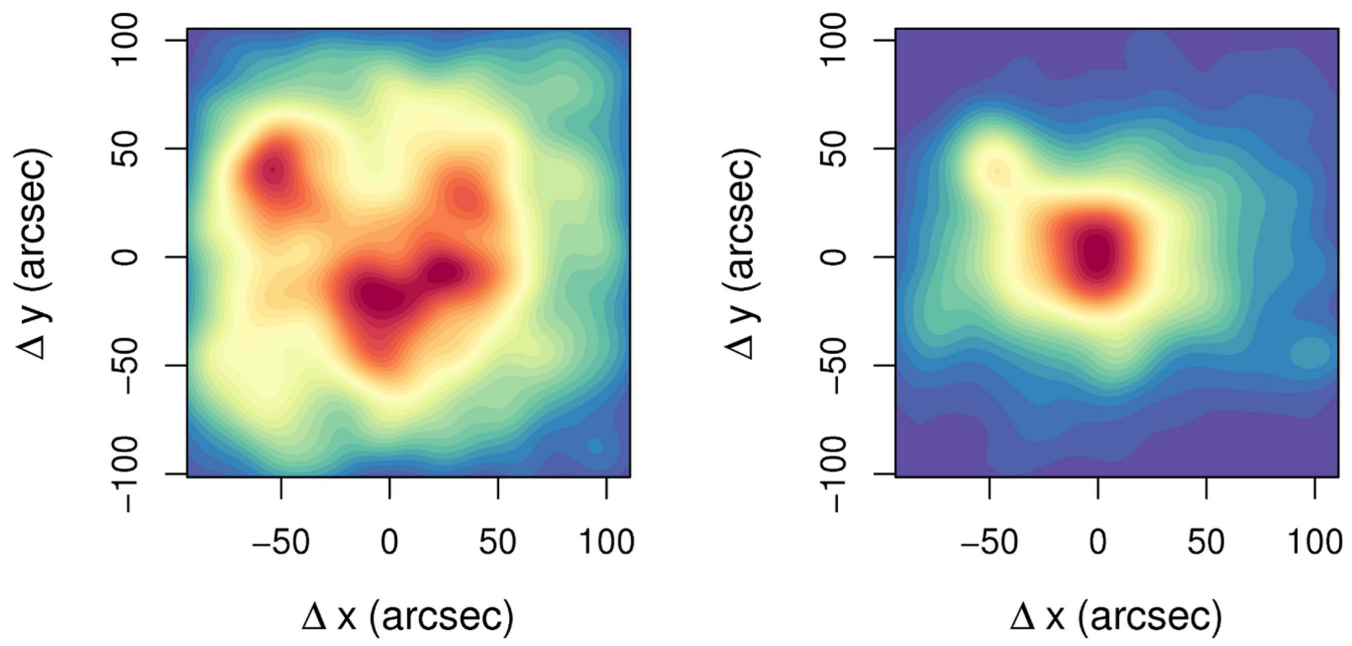

Figure 26. Spatial distributions in UGC 9799 for the extreme-MP clusters (left panel) and the extreme-MR clusters (right panel), shown as smoothed isocontour maps.
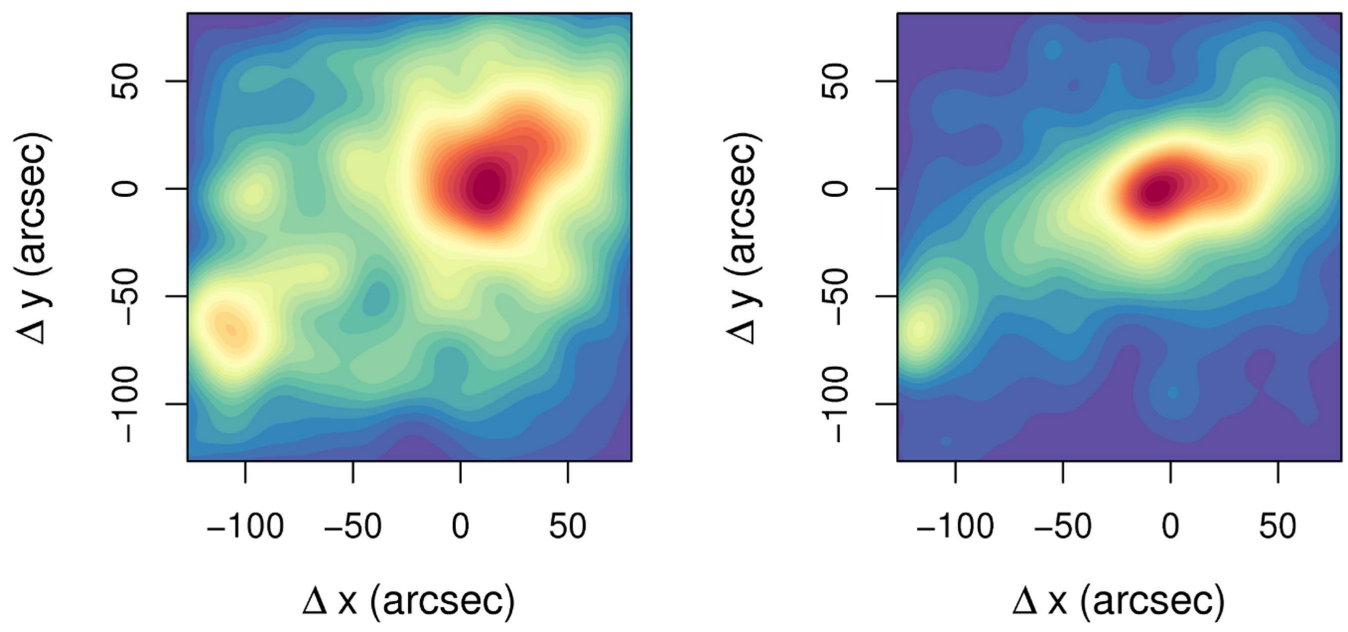

Figure 27. Spatial distributions in UGC 10143 for the EMP clusters (left panel) and the EMR clusters (right panel).
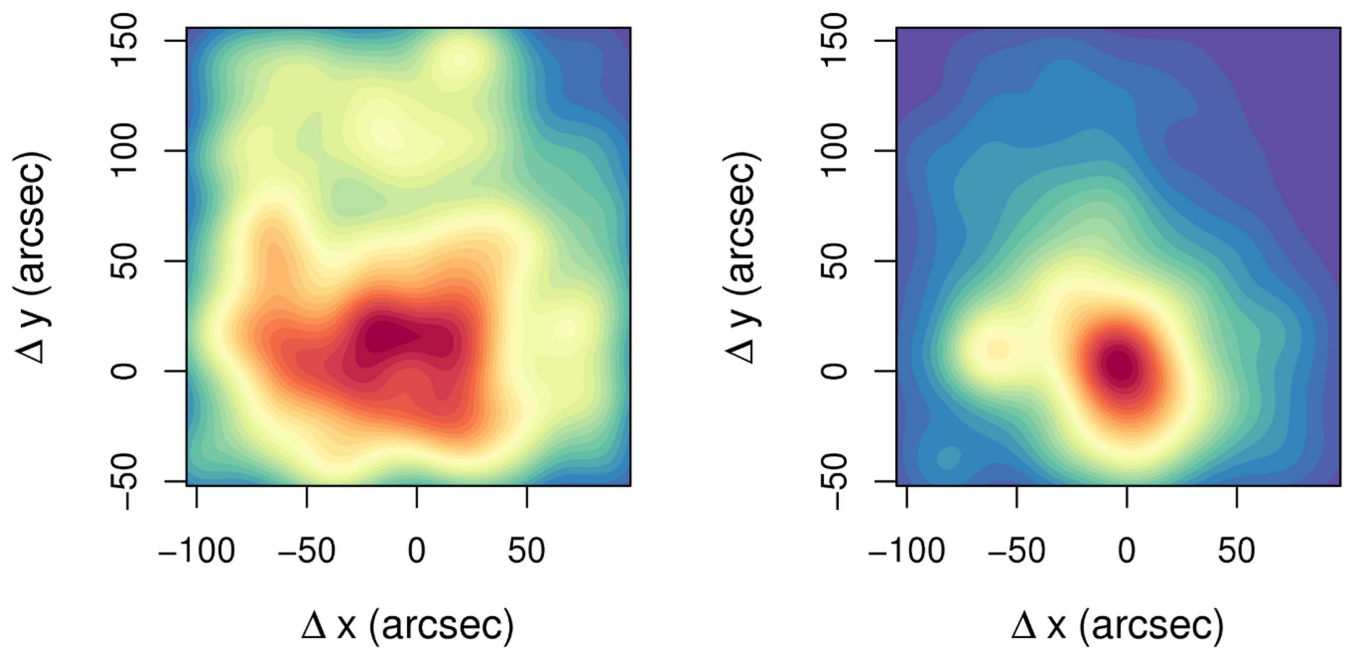

Figure 28. Spatial distributions in NGC 4889 for the EMP clusters (left panel) and the EMR clusters (right panel).

The halo light profile in $\mu_{V}$ as given in Pahre (1999) is shown as the dotted line in Figure 25; its slope matches the outer part of the EMR clusters well, although all parts of the GC distribution are very much shallower than the halo light for $R<30^{\prime \prime}$ (this radius is very nearly equal to $R_{\text {eff }}$ for the $\mu_{V}$ profile). Neither metallicity component follows a simple power law well, but in rough terms, the outer regions can be described as $\sigma(\mathrm{EMP}) \sim R^{-0.7}$ and $\sigma(\mathrm{EMR}) \sim R^{-1.8}$, a difference at least as large as we found for NGC 6166 and the other BCGs. 

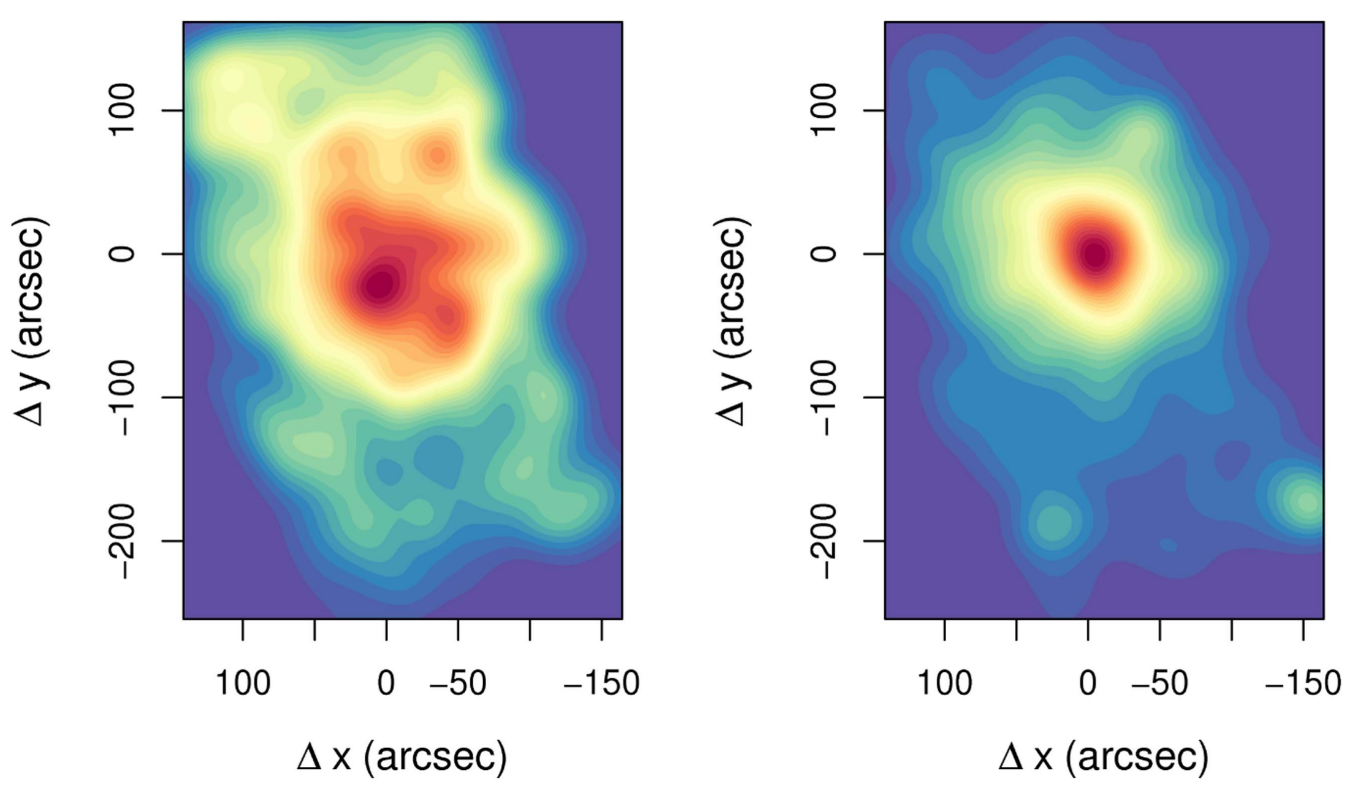

Figure 29. Spatial distributions in NGC 4874 for the EMP clusters (left panel) and the EMR clusters (right panel).

Table 4

Radial Profiles

\begin{tabular}{|c|c|c|c|c|c|c|c|}
\hline Galaxy & $\begin{array}{c}R_{e}(\mathrm{all}) \\
(\mathrm{kpc})\end{array}$ & $\begin{array}{l}R_{e}(\mathrm{EMP}) \\
\quad(\mathrm{kpc})\end{array}$ & $\begin{array}{l}R_{e}(\mathrm{EMR}) \\
\quad(\mathrm{kpc})\end{array}$ & $n$ (all) & $n$ (EMP) & $n(\mathrm{EMR})$ & Note \\
\hline NGC 4874 & 122 & 203 & 47 & 1.5 & 1.9 & 1.2 & Cho et al. (2016) \\
\hline NGC 4889 & 110 & 214 & 44 & 2.6 & 1.7 & 1.7 & this paper \\
\hline UGC 9799 & 61 & 80 & $\cdots$ & 1.4 & 1.0 & $\left(R^{-1.4}\right)$ & this paper \\
\hline UGC 10143 & 114 & 120 & $\ldots$ & 2.0 & 1.5 & $\left(R^{-1.2}\right)$ & this paper \\
\hline
\end{tabular}

We note that in all cases above, the halo-light radial profiles shown in the figures have been corrected for their ellipticity (see below) as $r_{\text {circ }}=a \sqrt{1-\epsilon}$, as described in Paper II.

\subsection{Azimuthal Distributions}

Another way in which the MP and MR subcomponents may reveal structural differences is in the ellipticity $\epsilon$ of their azimuthal distribution. In NGC 6166, we found that the mean $\epsilon$ for the EMR clusters matched the halo light, but the EMP clusters were more nearly spherically distributed. For the BCGs in this study, the contour maps described in the previous section show that this feature appears to be more general. As in Paper II, the method of moments (McLaughlin et al. 1994) is used to quantify the mean ellipticities $\epsilon$ of the subsystems.

For UGC 9799, clusters in the radial range $10^{\prime \prime}<R<100^{\prime \prime}$ were used. For the EMP component, $\epsilon=0.13 \pm 0.08$ and $\theta=25^{\circ} \pm 60^{\circ} \mathrm{E}$ of $\mathrm{N}$ for the position angle of the major axis; while for the EMR component $\epsilon=0.39 \pm 0.08, \theta=38^{\circ} \pm 5^{\circ}$. For all GCs combined, $\epsilon=0.37 \pm 16, \theta=41^{\circ} \pm 4^{\circ}$. For the halo light, Donzelli et al. (2011) give $\epsilon=0.35, \theta=35^{\circ} .5$ for the outer region (the more relevant comparison here). Clearly, the EMR clusters fairly accurately follow the halo light in both azimuthal and radial terms, while the EMP clusters follow a distribution that is scarcely distinguishable from spherical.

For UGC 10143, the results for GCs in the range $10^{\prime \prime}<R<70^{\prime \prime}$ are $\epsilon=0.19 \pm 0.15$ and $\theta=21^{\circ} \pm 62^{\circ}$ for the EMP sample; $\epsilon=0.51 \pm 0.23, \theta=11^{\circ} \pm 11^{\circ}$ for the EMR sample; and finally, for all GCs combined, $\epsilon=0.40 \pm$ $0.09, \theta=22^{\circ} \pm 10^{\circ}$. The ellipticity of the halo light increases rather markedly with $R$ (Donzelli et al. 2011), and to trace this out, we carried out our own isophotal mapping using stsdas/ ellipse on the $F 475 W$ image. Over $10^{\prime \prime}-70^{\prime \prime}, \epsilon_{F 475 W}$ increases from 0.27 to 0.47 , but the position angle stays nearly constant at $\theta \simeq 14^{\circ}$. Again, the EMR component matches the halo light within the uncertainties of the solution. An interesting additional feature of the EMP clusters is that their distribution appears to be somewhat asymmetric, with more of them spread to the upper right (northeast) in Figure 27; without having wider-field data to draw on, it is difficult to speculate what the cause might be.

Comparable studies for other galaxies of their azimuthal distributions for the MP and MR populations separately with comparable sample sizes are rare, but these other studies confirm a consistent pattern for the MP population to be distributed roughly spherically, while the MR population closely follows the halo light; see Geisler et al. (1996), Lee et al. (2008), Bassino et al. (2006), Harris (2009b), and Escudero et al. (2015) among others.

\subsection{Total Populations and Specific Frequencies}

In UGC 9799, the total population of GCs out to $R=150^{\prime \prime}$ (the limit of the ACS field of view, as well as the limit of the halo-light photometry) is $n=9650 \pm 190$ brighter than $I=27.0$ from integration of the radial profile. This limit is 0.12 mag fainter than the GCLF peak (Paper I) and thus should include $54 \%$ of the total over all luminosities assuming the GCLF is Gaussian in number per unit magnitude, and using the parameters from Paper I. We therefore obtain $N_{\text {tot }}=18000 \pm 400$ for $R<150^{\prime \prime}(110 \mathrm{kpc})$. Integrating the 
light profile (Seigar et al. 2007) to the same radius gives a total integrated magnitude $R_{\text {tot }}=12.55$, which corresponds to $M_{V}^{T} \simeq-22.85$ assuming a mean color $(V-R)=0.6$ for giant early-type galaxies. The specific frequency of the $\mathrm{GC}$ system is then $S_{N}=N_{\text {tot }} \cdot 10^{0.4\left(M_{V}^{T}+15\right)}=13.0 \pm 0.3$. If we were to choose to integrate the entire GC system profile all the way to the WFC3 field at $R=300 \mathrm{kpc}$, the result would be $N_{\text {tot }} \simeq 22,000$ clusters, but this estimate is much more uncertain.

For UGC 10143, if again we restrict the calculation conservatively to the ACS field of view $\left(R<155^{\prime \prime}\right)$, the total number of GCs with $I<27.0$ is $n=6450 \pm 100$, which translates into $N_{\text {tot }}=12500 \pm 200$ over all magnitudes. Integration of the halo light (Donzelli et al. 2011) into the same radius gives $R_{\mathrm{tot}}=12.43, M_{V}^{T} \simeq-22.96$. Finally then, $S_{N}=8.2 \pm 0.1$ to that radius. The more risky extrapolation of the GC profile out to the WFC3 field limit at $R=370^{\prime \prime}$ $(270 \mathrm{kpc})$ would give $N_{\text {tot }} \simeq 20,000$. The UGC $9799 \mathrm{GC}$ system is therefore relatively richer than for UGC 10143, but the specific frequencies of both are in the range observed for other BCGs (e.g., Harris et al. 2013).

For NGC 4889, the profile integration gives $n=8080 \pm 120$ clusters brighter than $F 814 W=26.5\left(M_{I}=-8.53\right)$; this limiting magnitude is very near the GCLF peak point. We therefore double that number to $N_{\text {tot }} \simeq 16000 \pm 250$, which when combined with $M_{V}^{T}=-23.65$ gives a specific frequency of $S_{N}=5.5 \pm 0.1$. This galaxy is very luminous, but relative to its size, it does not have an exceptionally populous cluster system in the more typical BCG range.

The story is somewhat different and more complex for the central Coma cD NGC 4874: Peng et al. (2011) estimate $N_{\text {tot }}=23000 \pm 700$ GCs excluding the more extended IGC population. When we use $M_{V}=-23.46$, as did Harris et al. (2009), we obtain $S_{N}=9.5 \pm 0.3$. If instead $N_{\text {tot }}$ is normalized to the entire luminosity profile out to a much larger radius of $520 \mathrm{kpc}$, then $S_{N}$ decreases markedly to $3.7 \pm 0.1$ (Peng et al. 2011); but since the IGC clusters outnumber the "intrinsic" GCs that are more clearly associated with the galaxy itself, this lower specific frequency is perhaps more of a statement about $S_{N}$ (IGC). In this respect, it is worth noting that Durrell et al. (2014) find $S_{N}=2.8 \pm 0.7$ for the Virgo cluster GCs in their entirety, of which a large fraction are IGCs, and less than a quarter are from M87 itself.

\section{DISCUSSION}

For extremely broad and nearly featureless MDFs such as we find in NGC 6166 (Paper II), UGC 9799, and UGC 10143, the imposition of a bimodal Gaussian numerical model, or any simplistic multimodal fitting process, begins to look increasingly arbitrary. Transformation of their CDFs back into the underlying metallicity distribution by way of Equation (1) yields a similarly broad unimodal MDF. We stress that this is a different issue than the one raised by Yoon et al. (2006), who proposed that an intrinsically unimodal MDF could be translated into the bimodal CDF seen in many galaxies because of the nonlinearity of the transformation (in their case, specifically the $(g-z)$ index, which, as noted above, is among the most nonlinear of the indices in common use). Here, we are essentially discussing the reverse: a CDF that is already smooth and unimodal cannot have come from an intrinsically bimodal MDF, because $(g-I) \rightarrow[\mathrm{Fe} / \mathrm{H}]$ is (mildly) nonlinear in the sense that it will produce a slightly more compressed
MDF rather than one that is more spread out. The same argument would apply to any of the other color indices used in other papers, such as $(g-z),(g-i),(V-I),(B-I)$, $\left(C-T_{1}\right)$, and others.

The MDF we observe today is the visible outcome of a rapid sequence of individual GC-forming events that took place within many halos along a hierarchical merger tree. For a galaxy at the BCG scale, thousands of such halos take part in this sequence at high redshift, each bringing in its own partially enriched gas. For a complex enough chain and a large enough number of halos, the end product might be expected to approach a continuous MDF, stretching from the most metalpoor GCs at an epoch where significant enrichment had not yet occurred, to the last major rounds of GC formation at roughly solar metallicity. In these supergiant cases, it is evident that the merging halos over their full range of masses contained enough gas to form large numbers of GCs at every metallicity from $[\mathrm{Fe} / \mathrm{H}] \sim-1.5$ up to above solar abundance, filling in every part of the MDF. At later times, individual accretions of small satellites continue that will add mostly to the metal-poor GC population in the outer halo.

Rather than attempting to reverse-engineer the formation events from properties of the color distributions and radial distributions, it would ultimately be preferable to move forward from a physics-based formation model to generate true model MDFs and GC spatial distributions that can then be compared with the observed cases. Early steps in this direction include Kravtsov \& Gnedin (2005), Griffen et al. (2010), Muratov \& Gnedin (2010), Tonini (2013), Li \& Gnedin (2014), and Li et al. (2016), but each of these papers uses particular simplifying prescriptions for the formation of GCs within the halos in the merger tree. Nevertheless, for galaxies at the highest masses, the outcome MDF in the models is broader and seen to approach a continuous distribution (Li \& Gnedin 2014).

An interesting result emerging from the BCGs, however, is that there are still notable differences in their MDFs even at these very highest galaxy masses. For M87 and NGC 4889, the MDFs still show a clearly bimodal form, while others (NGC 4874, NGC 6166) have MP and MR modes that have begun to merge, and in others (UGC 9799, UGC 10143) the MDF from the raw observations is smooth and unimodal, and the GMMfitted $D$-values fall below the threshold $D \simeq 2$. Nevertheless, in strict numerical terms, a bimodal Gaussian deconstruction matches all these cases extremely well. We emphasize, however (see again Section 2), that this model for the MDF is primarily a convenient description of its first-order features, emerging from what is intrinsically a continuous process of cluster formation. The overall appearance of the MDF is governed primarily by the internal dispersions $\sigma_{1}, \sigma_{2}$ of the MP and MR modes and thus the amount of overlap between the modes. By contrast, a near-uniform result we find is that the MP and MR mode centers $\mu_{1}, \mu_{2}$ in all the BCGs remain separated by very similar amounts $\Delta[\mathrm{Fe} / \mathrm{H}]=(0.81 \pm 0.04)$ dex, even though their dispersions may differ strongly.

Perhaps the most interesting feature is connected with the MP and MR relative numbers $\left(p_{1}, p_{2}\right)$. These supergiants contain roughly equal numbers of $\mathrm{MP}$ and MR clusters, but there are still striking differences such as in UGC 10143, where there are relatively few MR clusters at all radii, even in much of the inner region where the numbers of accreted MP clusters should have been small. This is, perhaps, an indication that the numbers of major gas-rich mergers from the large metal- 
enriched halos that would have produced the metal-richer GCs may differ strongly between different BCGs (see, e.g., Burke \& Collins 2013; Lidman et al. 2013). Other possibilities are that by the time such mergers occurred, the gas was already either largely converted into stars or heated to the point where less metal-rich GC formation could occur.

Our material strongly supports the identification of the metal-richer GCs with the halo light of their parent galaxies: their spatial distributions are similar in both radial and azimuthal distributions, and their overall spatial structure is consistently smoother and more regular than the MP clusters, like the galaxy light. Subdividing the sample into the EMP and EMR subgroups helps to emphasize their distinctive spatial distributions. This material adds to the similar evidence in other large galaxies (see Paper II), pointing to the conclusion that the MR clusters formed along with the main stellar population of the galaxy. By contrast, the EMP clusters consistently follow a distribution that in power-law terms $\sigma_{\mathrm{cl}} \sim R^{-\alpha}$ is shallower by 0.8-1.0 dex than the EMR clusters, very different from the structure of the halo light and fairly close to an isothermal form similar to the dark-matter halos.

The relation between gas metallicity and host galaxy mass (see Muratov \& Gnedin 2010; Li \& Gnedin 2014) strongly suggests that the MP GCs formed within small very metal-poor dwarfs. These GCs could therefore have accumulated either extremely early in the hierarchical chain, before the major body of the galaxy had fully assembled; or from accreted dwarf satellites that may come in at any later time. By contrast, the MR GCs should form within much bigger halos with more enriched gas. The importance of accretion in the buildup of the MDF that is observed today was first pointed out by Côté et al. $(1998,2000)$ and has frequently been discussed in the later literature (for comprehensive recent discussions of the relative importance of accretions, see Ferrarese et al. 2016; Kartha et al. 2016). BCGs are in highly privileged locations at the dynamical centers of rich galaxy clusters, and thus may gain the most from satellite accretions. The importance of their growth by dissipationless (dry) merging especially for redshifts $z<1$ has been emphasized in numerous recent discussions (e.g., Laporte et al. 2013; Liu et al. 2013; Laporte \& White 2015; Oliva-Altamirano et al. 2015; Oogi et al. 2016, among many others). The individual satellites may contribute both their own GCs and their stripped nuclei, which are structurally similar to luminous GCs. For example, Ferrarese et al. (2016) calculate that almost $40 \%$ of the GCs in the core region of the Virgo cluster around M87 may come from former satellites. A potentially related pattern that may be emerging from our BCG study is that the relative dominance of the MP clusters increases markedly beyond $R \gtrsim 4 R_{\text {eff }}$. This rough transition may mark the characteristic radius beyond which late accretions of metal-poor satellites dominate the GC population.

\section{SUMMARY}

Continuing our series of studies of the extraordinarily rich GCSs around BCGs with the HST ACS and WFC3 cameras, we present new comprehensive photometric analyses of the GC systems around UGC 9799 and UGC 10143, along with comparison data for the Coma supergiants NGC 4874 and NGC 4889. Our principal findings are these:

1. The GC systems in all these galaxies are, as expected, extremely populous, yielding total populations of anywhere from 12,000 to 23,000 clusters within galactocentric radii $\lesssim 120 \mathrm{kpc}$. Extrapolation to larger radii might almost double these totals, although the presence of asyet unknown numbers of intragalactic GCs will come into play there.

2. The color distribution (CDF) of the GCs has been measured in the $(F 475 W-F 814 W) \simeq(g-I)$ color index. In all the BCGs the CDF is broad, nominally unimodal, and skewed (asymmetric). Nevertheless, a simple bimodal Gaussian deconstruction continues to match the CDF very well, as it has for most smaller galaxies. The primary difference between these BCGs and smaller galaxies is that the intrinsic dispersions of the MP and MR modes become significantly higher, forcing the two modes to overlap heavily.

3. The broad, near-continuous form of the CDF implies that the intrinsic MDF must also be broad and unimodal, because the transformation from color to metallicity is only slightly nonlinear and acts in the direction of making the MDF slightly more compressed than the CDF.

4. Of the four galaxies discussed here, the Coma giants NGC 4874 and NGC 4889 show an MMR along the MP sequence, in the conventional sense that the blue sequence becomes systematically redder at higher luminosity. The heavy-element abundance scaling with GC mass is $Z \sim M^{0.25}$, which was also the case for NGC 6166 (Paper II). For UGC 10143, no detectable trend are detected along either the red or blue sequences. For UGC 9799 , the results are uncertain, with either a zero or positive slope along the blue sequence not ruled out. These results do not appear to support a simple selfenrichment model during GC formation, and instead may point to the need for some form of pre-enrichment in the most massive GCs driven by environmental differences at the time of formation.

5. The relative numbers of MP and MR clusters within these supergiants differ significantly in detail, with at least one case (UGC 10143) where the MP clusters dominate at all radii. This result suggests that the relative importance of gas-rich mergers with large metal-enriched halos (which built the MR clusters) could have differed between BCGs.

6. The ratio $N(\mathrm{MP}) / N($ tot $)$ increases with radius in all these galaxies, particularly past $R \gtrsim 4 R_{\text {eff }}$. We suggest tentatively that this transition radius may mark the region outside which the MP clusters that came from late, discrete accretions of dwarf satellite galaxies are most important.

7. In all these BCGs, as in other large galaxies, the extremely metal-poor GCs follow a roughly spherical spatial distribution not far from the form $\sigma \sim R^{-1}$ that would characterize the dark-matter halo. By contrast, the most metal-rich GCs follow a more concentrated, smooth, and regular distribution that matches the halo light of the galaxy. The MR clusters are most likely to have formed along with the main stellar body of the galaxy.

Based on observations made with the NASA/ESA HST, obtained at the Space Telescope Science Institute, which is operated by the Association of Universities for Research in Astronomy, Inc., under NASA contract NAS 5-26555. W.E.H. and G.M.E. acknowledge financial support from NSERC (Natural Sciences and Engineering Research Council of 
Canada). J.B. acknowledges financial support from program HST-AR-13908.001-A provided by NASA through a grant from the Space Telescope Science Institute. O.Y.G. was supported in part by NASA through grant NNX12AG44G, and by NSF through grant 1412144. D.G. gratefully acknowledges support from the Chilean BASAL Centro de Excelencia en Astrofísica y Tecnologías Afines (CATA) grant PFB06/2007.

Facility: HST (ACS, WFC3).

\section{REFERENCES}

Alamo-Martínez, K. A., Blakeslee, J. P., Jee, M. J., et al. 2013, ApJ, 775, 20 Alves-Brito, A., Hau, G. K. T., Forbes, D. A., et al. 2011, MNRAS, 417, 1823 Andersen, M., Zinnecker, H., Moneti, A., et al. 2009, ApJ, 707, 1347 Arnold, J. A., Romanowsky, A. J., Brodie, J. P., et al. 2011, ApJL, 736, L26 Baade, W. 1958, RA, 5, 3

Bailin, J., \& Harris, W. E. 2009, ApJ, 695, 1082

Balmaverde, B., Capetti, A., \& Grandi, P. 2006, A\&A, 451, 35

Barmby, P., \& Huchra, J. P. 1998, AJ, 115, 6

Barmby, P., Huchra, J. P., Brodie, J. P., et al. 2000, AJ, 119, 727

Bassino, L. P., Faifer, F. R., Forte, J. C., et al. 2006, A\&A, 451, 789

Bassino, L. P., Richtler, T., \& Dirsch, B. 2008, MNRAS, 386, 1145

Bertin, E., \& Arnouts, S. 1996, A\&AS, 117, 393

Blakeslee, J. P. 1999, AJ, 118, 1506

Blakeslee, J. P., Cho, H., Peng, E. W., et al. 2012, ApJ, 746, 88

Blakeslee, J. P., \& Tonry, J. L. 1995, ApJ, 442, 579

Blanton, E. L., Randall, S. W., Clarke, T. E., et al. 2011, ApJ, 737, 99

Blanton, E. L., Sarazin, C. L., \& McNamara, B. R. 2003, ApJ, 585, 227

Blom, C., Forbes, D. A., Brodie, J. P., et al. 2012a, MNRAS, 426, 1959

Blom, C., Spitler, L. R., \& Forbes, D. A. 2012b, MNRAS, 420, 37

Brodie, J. P., Romanowsky, A. J., Strader, J., et al. 2014, ApJ, 796, 52

Brodie, J. P., \& Strader, J. 2006, ARA\&A, 44, 193

Brodie, J. P., Usher, C., Conroy, C., et al. 2012, ApJL, 759, L33

Burke, C., \& Collins, C. A. 2013, MNRAS, 434, 2856

Buttiglione, S., Capetti, A., Celotti, A., et al. 2010, A\&A, 509, A6

Caldwell, N., \& Romanowsky, A. J. 2016, ApJ, 824, 42

Caldwell, N., Schiavon, R., Morrison, H., Rose, J. A., \& Harding, P. 2011, AJ, 141, 61

Cantiello, M., \& Blakeslee, J. P. 2007, ApJ, 669, 982

Cantiello, M., Blakeslee, J. P., Raimondo, G., et al. 2014, A\&A, 564, L3

Cardelli, J. A., Clayton, G. C., \& Mathis, J. S. 1989, ApJ, 345, 245

Cezario, E., Coelho, P. R. T., Alves-Brito, A., Forbes, D. A., \& Brodie, J. P. 2013, A\&A, 549, A60

Chies-Santos, A. L., Larsen, S. S., Cantiello, M., et al. 2012a, A\&A, 539, A54

Chies-Santos, A. L., Larsen, S. S., \& Kissler-Patig, M. 2012b, MNRAS, 427, 2349

Cho, H., Blakeslee, J. P., Chies-Santos, A. L., et al. 2016, ApJ, 822, 95

Chung, C., Yoon, S.-J., Lee, S.-Y., \& Lee, Y.-W. 2016, ApJ, 818, 201

Cockcroft, R., Harris, W. E., Wehner, E. M. H., Whitmore, B. C., \& Rothberg, B. 2009, AJ, 138, 758

Côté, P., Marzke, R. O., \& West, M. J. 1998, ApJ, 501, 554

Côté, P., Marzke, R. O., West, M. J., \& Minniti, D. 2000, ApJ, 533, 869

Dalla Bontà, E., Ferrarese, L., Corsini, E. M., et al. 2009, ApJ, 690, 537

Donato, D., Sambruna, R. M., \& Gliozzi, M. 2004, ApJ, 617, 915

Donzelli, C. J., Muriel, H., \& Madrid, J. P. 2011, ApJS, 195, 15

Durrell, P. R., Côté, P., Peng, E. W., et al. 2014, ApJ, 794, 103

Durrell, P. R., Sarajedini, A., \& Chandar, R. 2010, ApJ, 718, 1118

Edwards, L. O. V., Hudson, M. J., Balogh, M. L., \& Smith, R. J. 2007, MNRAS, 379, 100

Escudero, C. G., Faifer, F. R., Bassino, L. P., Calderón, J. P., \& Caso, J. P. 2015, MNRAS, 449, 612

Faifer, F. R., Forte, J. C., Norris, M. A., et al. 2011, MNRAS, 416, 155

Falco, M., Hansen, S. H., Wojtak, R., et al. 2014, MNRAS, 442, 1887

Fan, Z., Ma, J., Zhou, X., \& Jiang, Z. 2010, PASP, 122, 636

Fensch, J., Mieske, S., Müller-Seidlitz, J., \& Hilker, M. 2014, A\&A, 567, A105

Ferrarese, L., Côté, P., Sánchez-Janssen, R., et al. 2016, ApJ, 824, 10

Forbes, D. A., Brodie, J. P., \& Grillmair, C. J. 1997, AJ, 113, 1652

Forbes, D. A., Spitler, L. R., Harris, W. E., et al. 2010, MNRAS, 403, 429

Forbes, D. A., Spitler, L. R., Strader, J., et al. 2011, MNRAS, 413, 2943

Forte, J. C., Faifer, F., \& Geisler, D. 2007, MNRAS, 382, 1947

Forte, J. C., Faifer, F. R., Vega, E. I., et al. 2013, MNRAS, 431, 1405

Foster, C., Forbes, D. A., Proctor, R. N., et al. 2010, AJ, 139, 1566
Gebhardt, K., \& Kissler-Patig, M. 1999, AJ, 118, 1526

Geisler, D., Lee, M. G., \& Kim, E. 1996, AJ, 111, 1529

Griffen, B. F., Drinkwater, M. J., Thomas, P. A., Helly, J. C., \& Pimbblet, K. A. 2010, MNRAS, 405, 375

Harris, W. E. 1987, ApJL, 315, L29

Harris, W. E. 2009a, ApJ, 699, 254

Harris, W. E. 2009b, ApJ, 703, 939

Harris, W. E., Blakeslee, J. P., Whitmore, B. C., et al. 2016, ApJ, 817, 58

Harris, W. E., \& Canterna, R. 1979, ApJL, 231, L19

Harris, W. E., Harris, G. L. H., \& Alessi, M. 2013, ApJ, 772, 82

Harris, W. E., Harris, G. L. H., Layden, A. C., \& Wehner, E. M. H. 2007, ApJ, 666, 903

Harris, W. E., Kavelaars, J. J., Hanes, D. A., Pritchet, C. J., \& Baum, W. A. 2009, AJ, 137, 3314

Harris, W. E., Morningstar, W., Gnedin, O. Y., et al. 2014, ApJ, 797, 128

Harris, W. E., Pritchet, C. J., \& McClure, R. D. 1995, ApJ, 441, 120

Harris, W. E., Spitler, L. R., Forbes, D. A., \& Bailin, J. 2010, MNRAS, 401, 1965

Harris, W. E., Whitmore, B. C., Karakla, D., et al. 2006, ApJ, 636, 90

Hartmann, L., Ballesteros-Paredes, J., \& Heitsch, F. 2012, MNRAS, 420, 1457

Hicks, A. K., Mushotzky, R., \& Donahue, M. 2010, ApJ, 719, 1844

Hoffer, A. S., Donahue, M., Hicks, A., \& Barthelemy, R. S. 2012, ApJS, 199,23

Howard, C. S., Pudritz, R. E., \& Harris, W. E. 2016, MNRAS, 461, 2953

Jennings, Z. G., Strader, J., Romanowsky, A. J., et al. 2014, AJ, 148, 32

Jordán, A., McLaughlin, D. E., Côté, P., et al. 2007, ApJS, 171, 101

Kartha, S. S., Forbes, D. A., Alabi, A. B., et al. 2016, MNRAS, 458, 105

Kartha, S. S., Forbes, D. A., Spitler, L. R., et al. 2014, MNRAS, 437, 273

Kim, H.-S., Yoon, S.-J., Sohn, S. T., et al. 2013, ApJ, 763, 40

Kinman, T. D. 1959, MNRAS, 119, 538

Kravtsov, A. V., \& Gnedin, O. Y. 2005, ApJ, 623, 650

Kubo, J. M., Stebbins, A., Annis, J., et al. 2007, ApJ, 671, 1466

Kundu, A., \& Whitmore, B. C. 2001, AJ, 121, 2950

Kundu, A., \& Zepf, S. E. 2007, ApJL, 660, L109

Lada, C. J., \& Lada, E. A. 2003, ARA\&A, 41, 57

Laporte, C. F. P., \& White, S. D. M. 2015, MNRAS, 451, 1177

Laporte, C. F. P., White, S. D. M., Naab, T., \& Gao, L. 2013, MNRAS, 435,901

Larsen, S. S., Brodie, J. P., Huchra, J. P., Forbes, D. A., \& Grillmair, C. J. 2001, AJ, 121, 2974

Larsen, S. S., Brodie, J. P., \& Strader, J. 2005, A\&A, 443, 413

Lee, M. G., \& Jang, I. S. 2016a, ApJ, 819, 77

Lee, M. G., \& Jang, I. S. 2016b, ApJ, 831, 108

Lee, M. G., Park, H. S., Kim, E., et al. 2008, ApJ, 682, 135

Li, H., \& Gnedin, O. Y. 2014, ApJ, 796, 10

Li, H., Gnedin, O. Y., Gnedin, N. Y., et al. 2016, arXiv:1608.03244

Lidman, C., Iacobuta, G., Bauer, A. E., et al. 2013, MNRAS, 433, 825

Liu, C., Peng, E. W., Jordán, A., et al. 2011, ApJ, 728, 116

Liu, F. S., Guo, Y., Koo, D. C., et al. 2013, ApJ, 769, 147

Łokas, E. L., Wojtak, R., Gottlöber, S., Mamon, G. A., \& Prada, F. 2006 , MNRAS, 367, 1463

Ma, J., Wu, Z.-Y., Zhang, T.-M., et al. 2013, RAA, 13, 399

Machado, R. E. G., \& Lima Neto, G. B. 2015, MNRAS, 447, 2915

Marín-Franch, A., \& Aparicio, A. 2002, ApJ, 568, 174

Marsakov, V. A., \& Suchkov, A. A. 1976, SvAL, 2, 148

Martel, A. R., Sparks, W. B., Allen, M. G., Koekemoer, A. M., \& Baum, S. A. 2002, AJ, 123, 1357

Mayall, N. U. 1946, ApJ, 104, 290

McConnachie, A. W., Irwin, M. J., Ibata, R. A., et al. 2009, Natur, 461, 66

McLaughlin, D. E., Harris, W. E., \& Hanes, D. A. 1994, ApJ, 422, 486

Melena, N. W., Massey, P., Morrell, N. I., \& Zangari, A. M. 2008, AJ, 135,878

Mieske, S., Jordán, A., Côté, P., et al. 2006, ApJ, 653, 193

Mieske, S., Jordán, A., Côté, P., et al. 2010, ApJ, 710, 1672

Monachesi, A., Bell, E. F., Radburn-Smith, D. J., et al. 2016, MNRAS, 457, 1419

Morgan, W. W. 1956, PASP, 68, 509

Muratov, A. L., \& Gnedin, O. Y. 2010, ApJ, 718, 1266

Neilsen, E. H., Jr., \& Tsvetanov, Z. I. 1999, ApJL, 515, L13

Oliva-Altamirano, P., Brough, S., Jimmy, K.-Vy. T., et al. 2015, MNRAS, 449, 3347

Oogi, T., Habe, A., \& Ishiyama, T. 2016, MNRAS, 456, 300

Pahre, M. A. 1999, ApJS, 124, 127

Peacock, M. B., Zepf, S. E., Maccarone, T. J., \& Kundu, A. 2011, ApJ, 737, 5

Peng, E. W., Ferguson, H. C., Goudfrooij, P., et al. 2011, ApJ, 730, 23

Peng, E. W., Ford, H. C., \& Freeman, K. C. 2004, ApJS, 150, 367 
Peng, E. W., Jordán, A., Blakeslee, J. P., et al. 2009, ApJ, 703, 42

Peng, E. W., Jordán, A., Côté, P., et al. 2006, ApJ, 639, 95

Perrett, K. M., Bridges, T. J., Hanes, D. A., et al. 2002, AJ, 123, 2490

Rejkuba, M., Harris, W. E., Greggio, L., \& Harris, G. L. H. 2011, A\&A, 526, A123

Rejkuba, M., Harris, W. E., Greggio, L., et al. 2014, ApJL, 791, L2

Renzini, A., D’Antona, F., Cassisi, S., et al. 2015, MNRAS, 454, 4197

Rhode, K. L., \& Zepf, S. E. 2004, AJ, 127, 302

Sakari, C. M., \& Wallerstein, G. 2016, MNRAS, 456, 831

Schlafly, E. F., \& Finkbeiner, D. P. 2011, ApJ, 737, 103

Schneider, F. R. N., Izzard, R. G., de Mink, S. E., et al. 2014, ApJ, 780, 117

Searle, L., \& Zinn, R. 1978, ApJ, 225, 357

Seigar, M. S., Graham, A. W., \& Jerjen, H. 2007, MNRAS, 378, 1575

Sinnott, B., Hou, A., Anderson, R., Harris, W. E., \& Woodley, K. A. 2010, AJ, 140,2101

Spitler, L. R., Forbes, D. A., \& Beasley, M. A. 2008, MNRAS, 389, 1150

Stetson, P. B. 1987, PASP, 99, 191

Strader, J., Beasley, M. A., \& Brodie, J. P. 2007, AJ, 133, 2015

Strader, J., Brodie, J. P., Spitler, L., \& Beasley, M. A. 2006, AJ, 132, 2333

Strader, J., Romanowsky, A. J., Brodie, J. P., et al. 2011, ApJS, 197, 33
Thompson, L. A., \& Valdes, F. 1987, ApJL, 315, L35

Tonini, C. 2013, ApJ, 762, 39

Tovmassian, H. M., \& Andernach, H. 2012, MNRAS, 427, 2047

Usher, C., Forbes, D. A., Brodie, J. P., et al. 2012, MNRAS, 426, 1475

Usher, C., Forbes, D. A., Brodie, J. P., et al. 2015, MNRAS, 446, 369

Usher, C., Forbes, D. A., Spitler, L. R., et al. 2013, MNRAS, 436, 1172

VanDalfsen, M. L., \& Harris, W. E. 2004, AJ, 127, 368

Vanderbeke, J., West, M. J., De Propris, R., et al. 2014, MNRAS, 437, 1734

Villegas, D., Jordán, A., Peng, E. W., et al. 2010, ApJ, 717, 603

Wehner, E. M. H., Harris, W. E., Whitmore, B. C., Rothberg, B., \& Woodley, K. A. 2008, ApJ, 681, 1233

Wen, Z. L., Han, J. L., \& Liu, F. S. 2010, MNRAS, 407, 533

West, M. J., Jordán, A., Blakeslee, J. P., et al. 2011, A\&A, 528, A115

Wojtak, R., \& Lokas, E. L. 2010, MNRAS, 408, 2442

Woodley, K. A., Harris, W. E., Puzia, T. H., et al. 2010, ApJ, 708, 1335

Yoon, S.-J., Lee, S.-Y., Blakeslee, J. P., et al. 2011a, ApJ, 743, 150

Yoon, S.-J., Sohn, S. T., Lee, S.-Y., et al. 2011b, ApJ, 743, 149

Yoon, S.-J., Yi, S. K., \& Lee, Y.-W. 2006, Sci, 311, 1129

Zepf, S. E., \& Ashman, K. M. 1993, MNRAS, 264, 611

Zinn, R. 1985, ApJ, 293, 424 